
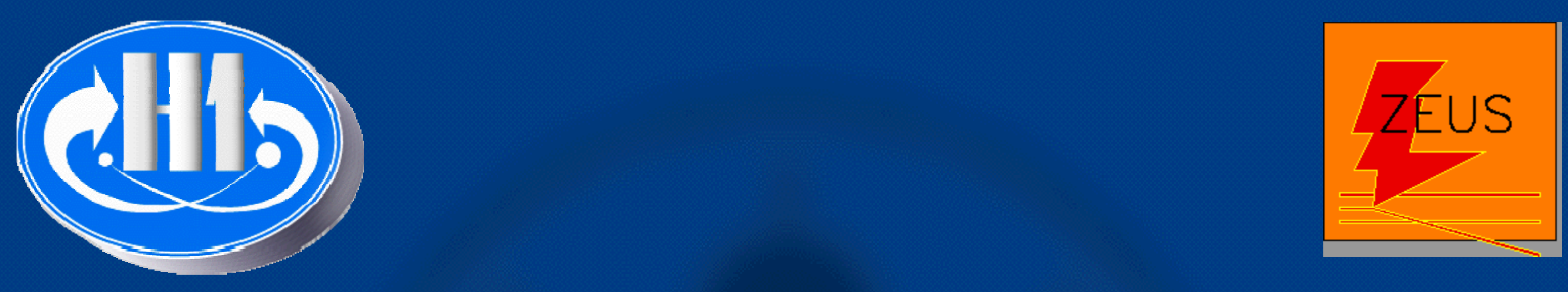

\title{
The Dynamics of
} Proton Structure: Recent Results from HERA

MHADRRPINOS 5

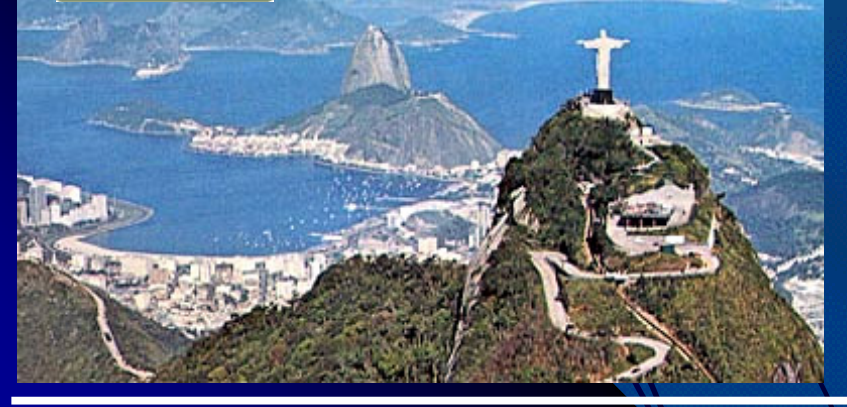

23 August, 2005 Tobias Haas Deutsches Elektronensynchrotron 


\section{This Talk}

- Introduction to HERA

- Standard model physics

- NC/CC electrons/positrons, $+/$ - helicities

- Very high $\mathrm{x}$ cross sections

- Heavy quarks

- $F_{2}, F_{3}, F_{2}{ }^{c}, F_{2}^{b}$

- Parton densities

$a_{s}(M Z)$

- EW parameters
- Beyond the standard model

- Quark compositeness

- Contact interactions

- Leptoquarks

- High $p_{\mathrm{T}}$ leptons

- Summary and conclusions 


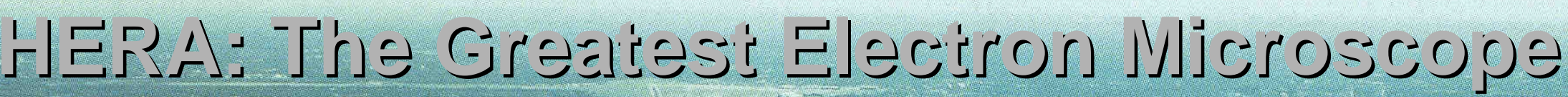

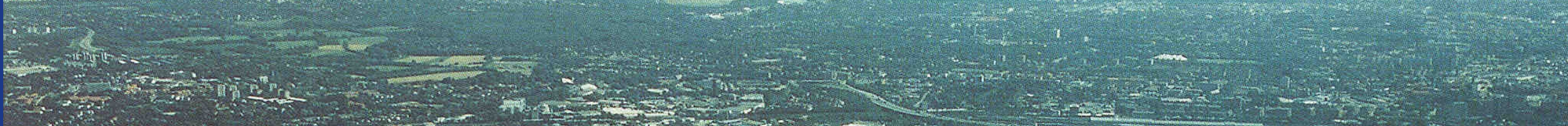

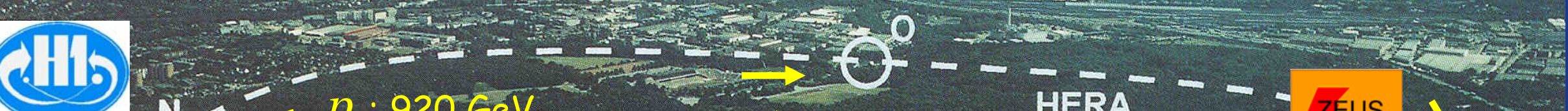

$$
\begin{aligned}
& \text { N }-P: 920 \mathrm{GeV}-e^{ \pm} \quad \text { HERA }- \text { ZEUS } \perp \\
& 1=2 e^{ \pm}: 27.5 \mathrm{GeV} \\
& \text { s } \\
& \text { if }
\end{aligned}
$$

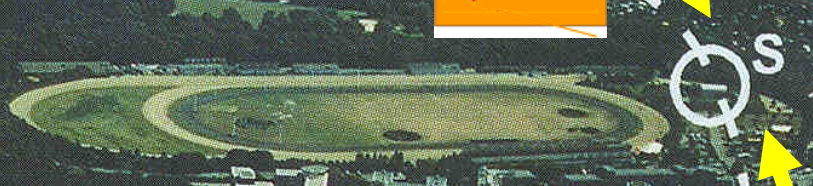

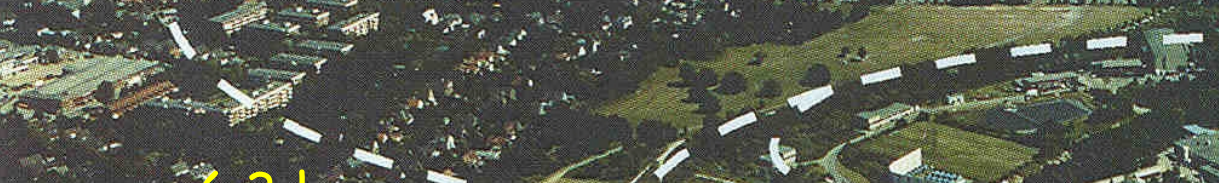

-

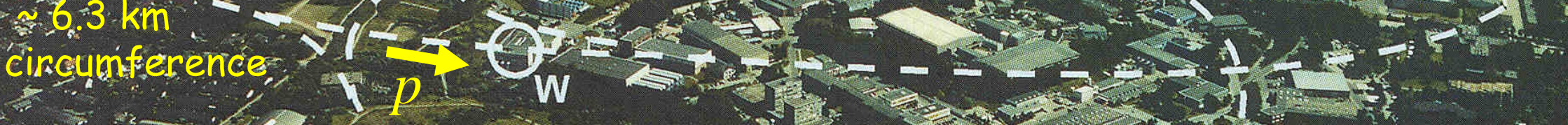

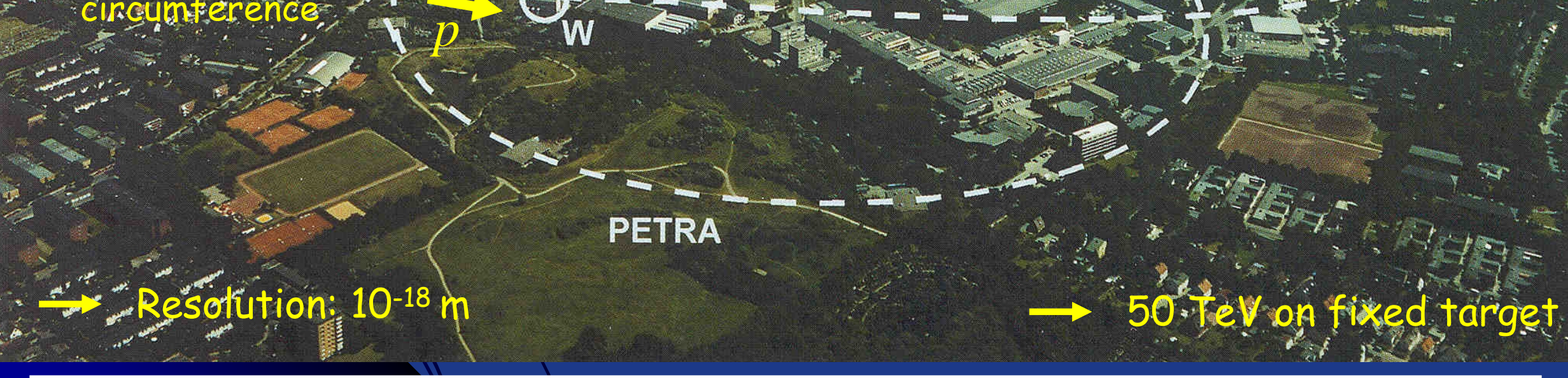




\section{Luminosity, $\mathrm{e}^{ \pm}$and polarization = HERA II}

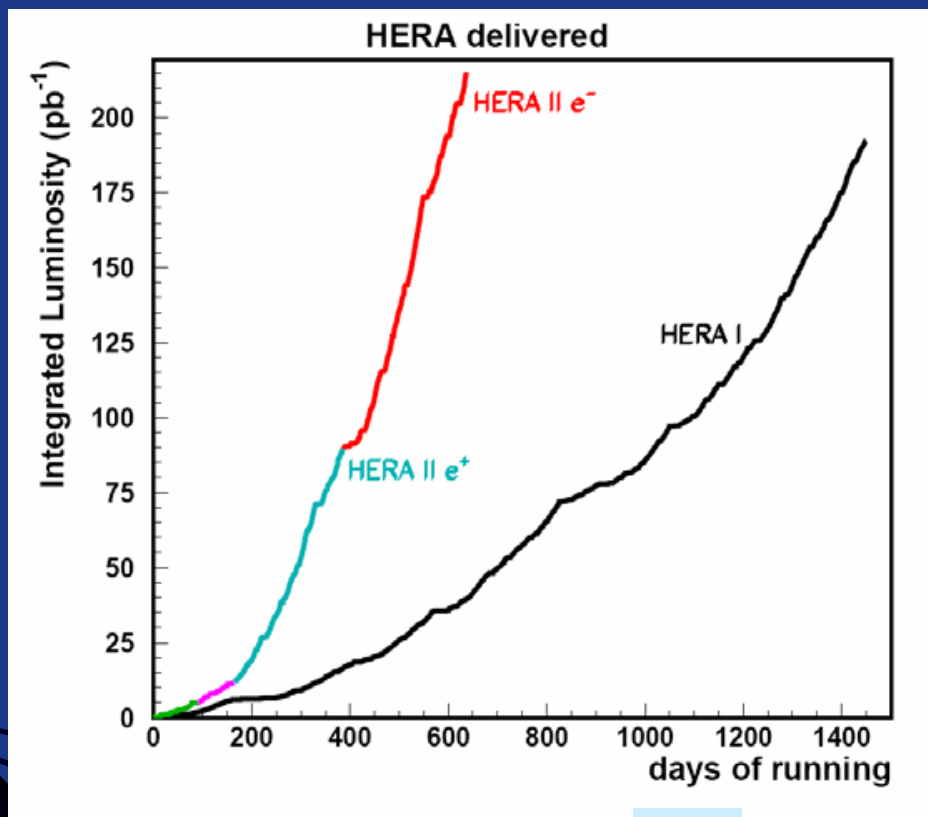

- > $200 \mathrm{pb}^{-1} /$ expt delivered

- Both lepton species

- Expect $~ 700 \mathrm{pb}^{-1} /$ expt by the end of HERA (2007)

- Polarization:

- $50 \%$ reached routinely now

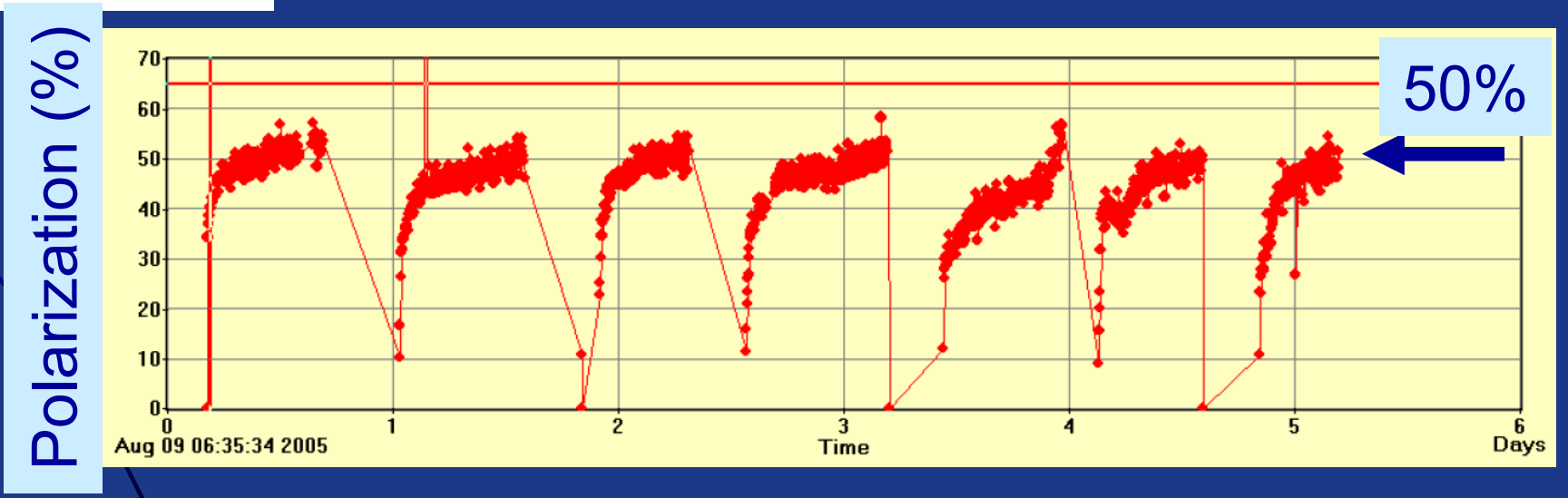




\section{DIS@HERA}
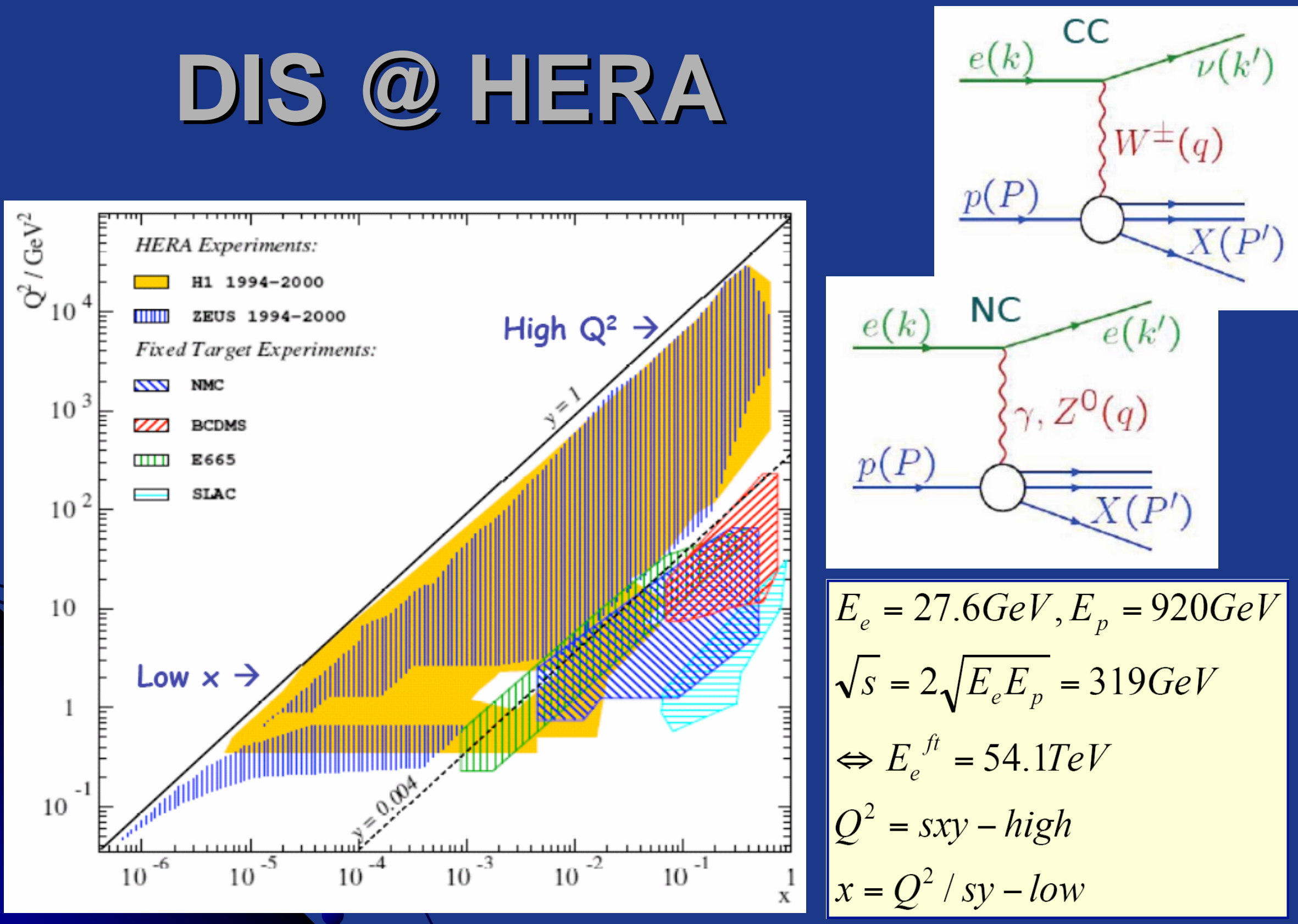


\section{Standard Model}


Neutral current $e^{+} p \rightarrow e^{+} X$

$\therefore$

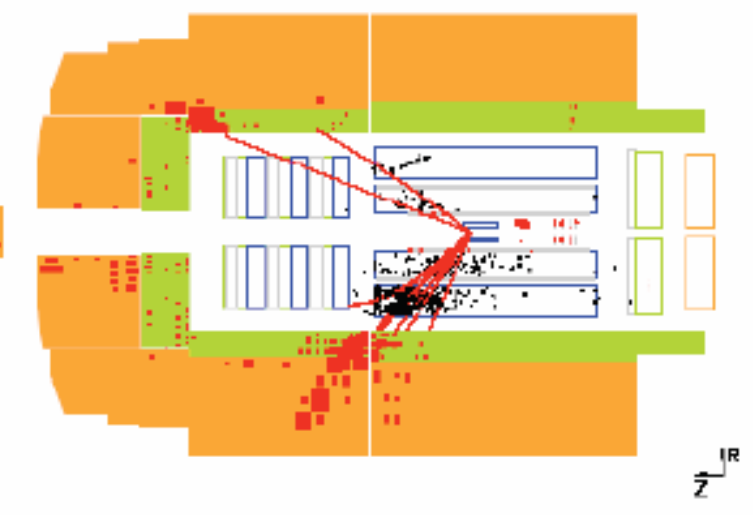
Neutral Currents

Charged current $e^{+} p \rightarrow \bar{v} X$

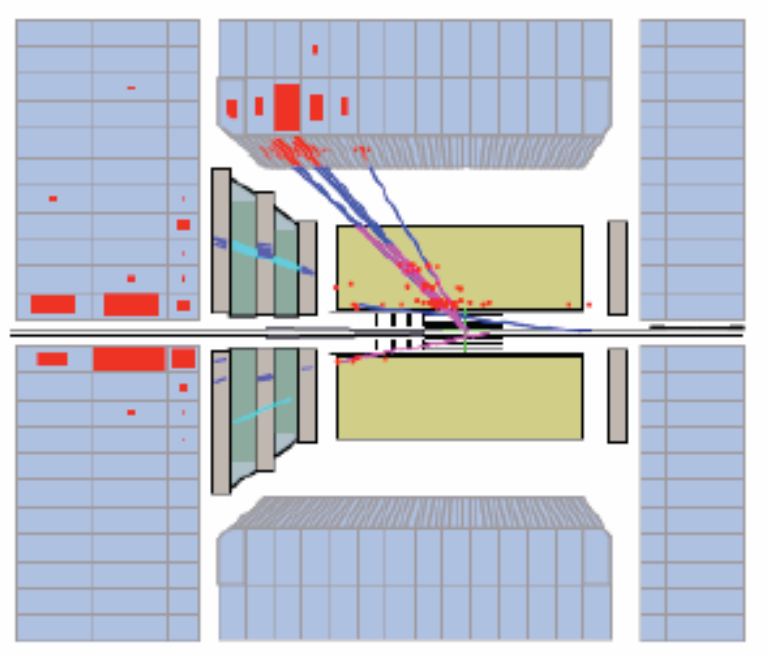

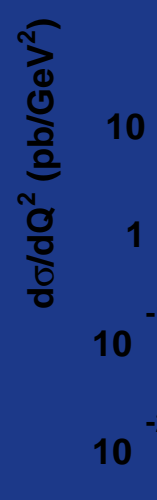

$10^{-3}$

$10^{-4}$

$10^{-5}$

10

$10^{-6}$

$10^{-7} \quad y<0.9$
H1 e'p NC 94-00

H1 e-p NC

ZEUS (prel.) $e^{+} p$ NC 99-00

ZEUS ēp NC 98-99

$S M e^{+} p$ NC (CTEQ6D)

SM e p NC (CTEQ6D)

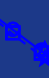

H1 e+p CC 94-00

H1 e p CC

ZEUS $e^{+} p$ CC 99-00

ZEUS e-p CC 98-99

$S M e^{+} p$ CC (CTEQ6D)

SM e־p CC (CTEQ6D)
Charged

Currents

$$
\sigma_{N C}^{ \pm} \approx \sigma_{C C}^{ \pm} \leftrightarrow Q^{2} \approx M_{Z}^{2} \approx 10^{4} \mathrm{GeV}^{2}
$$




\section{Charged Currents}

- SM expectation:

- $\sigma^{ \pm}\left(P_{e}\right)=\left(1 \pm P_{e}\right) \sigma^{ \pm}\left(P_{e}=0\right)$
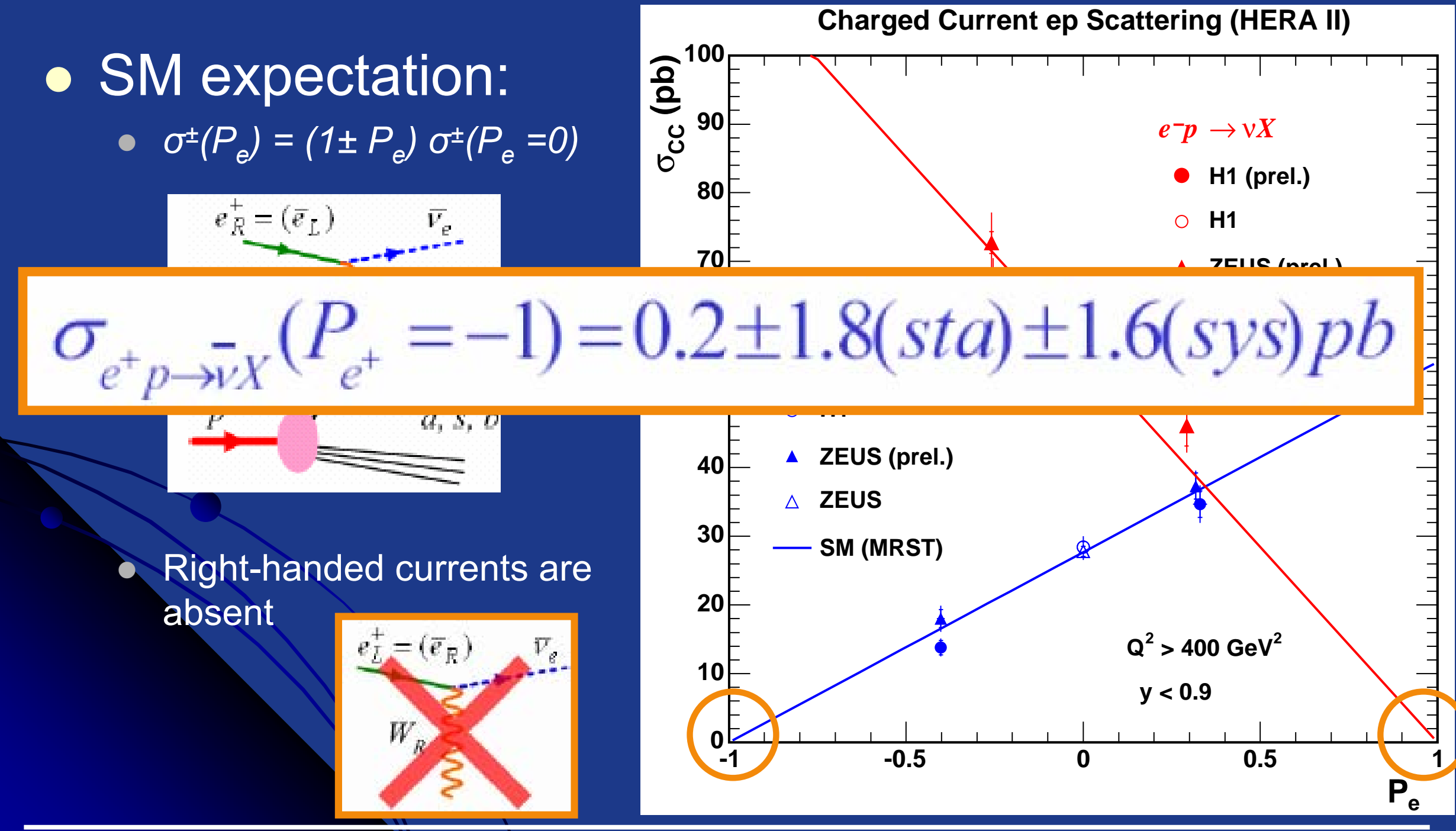


\section{Neutral Currents: The Expectation}

Neutral Current Cross Section

-- Polarisation dependence appears through the interference between $\gamma$ and $\mathrm{Z}$ boson exchanges

$$
\frac{d^{2} \sigma^{N C}\left(e^{ \pm} p\right)}{d x d Q^{2}}=\frac{2 \pi \alpha}{x Q^{2}}\left[H_{0}^{ \pm}+P \cdot H_{P}^{ \pm}\right]
$$

$\mathrm{H}_{0}$ : unpolarised structure function

$\mathrm{H}_{\mathrm{P}}$ : polarised structure function

-- Polarisation effect can be visible only at high $\mathrm{Q}^{2}$ region

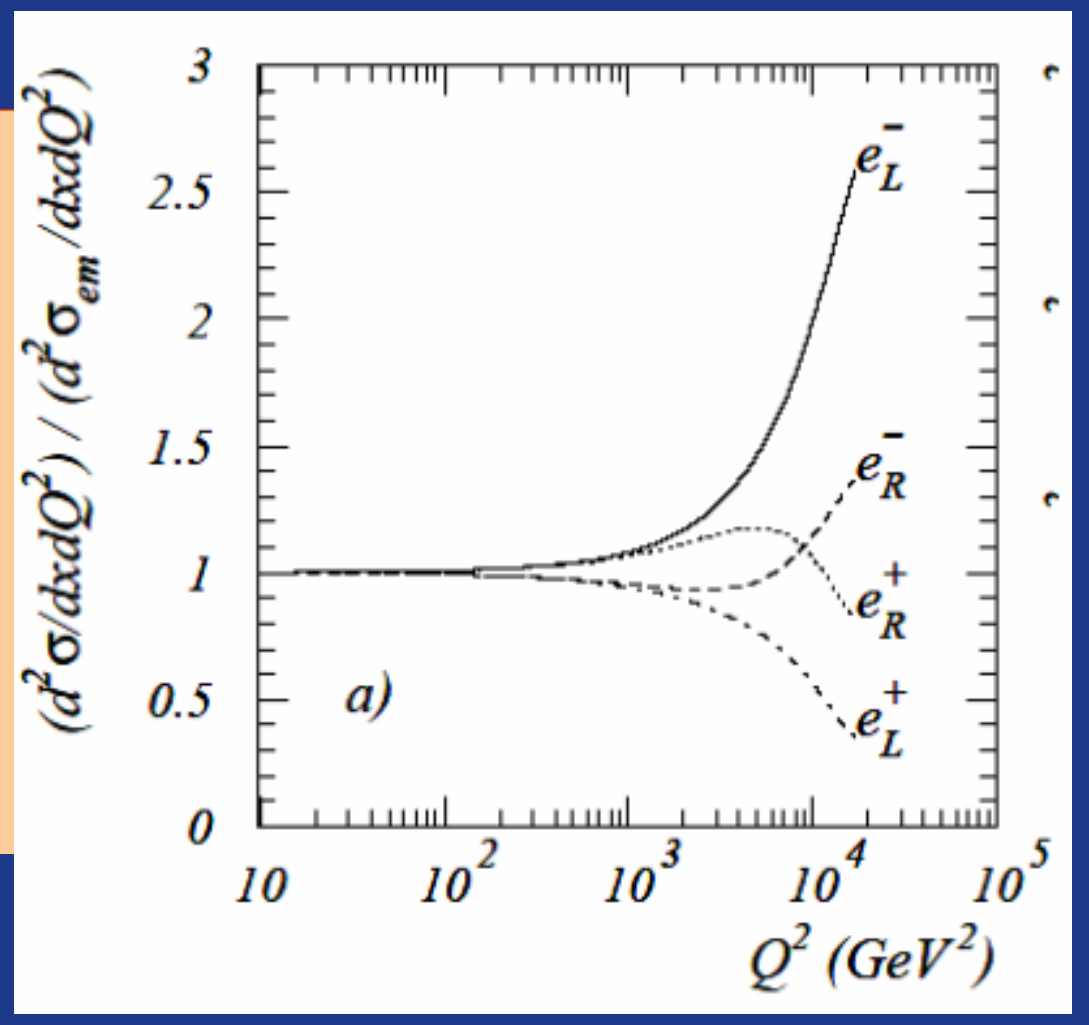




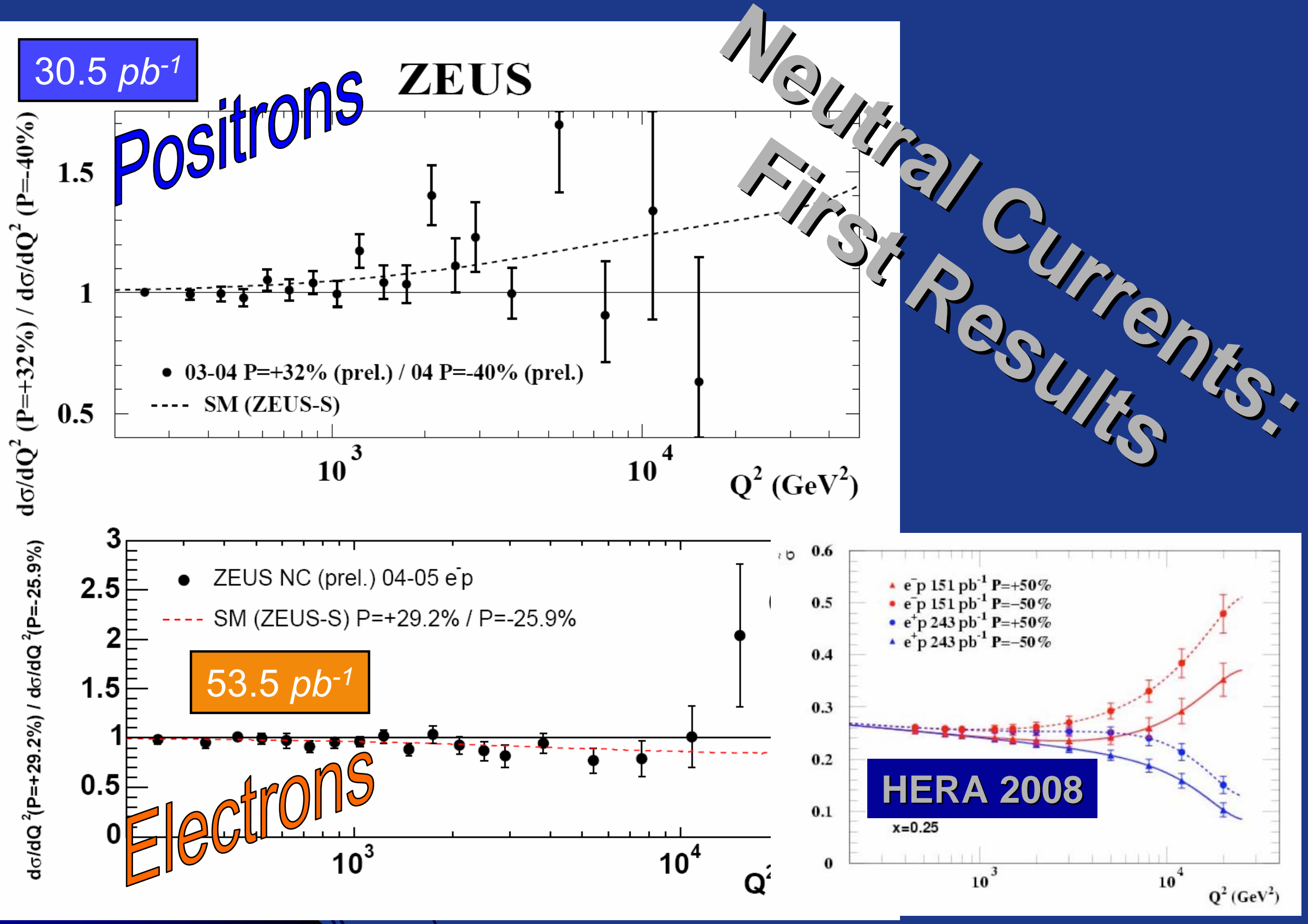




\section{What do we learn?}

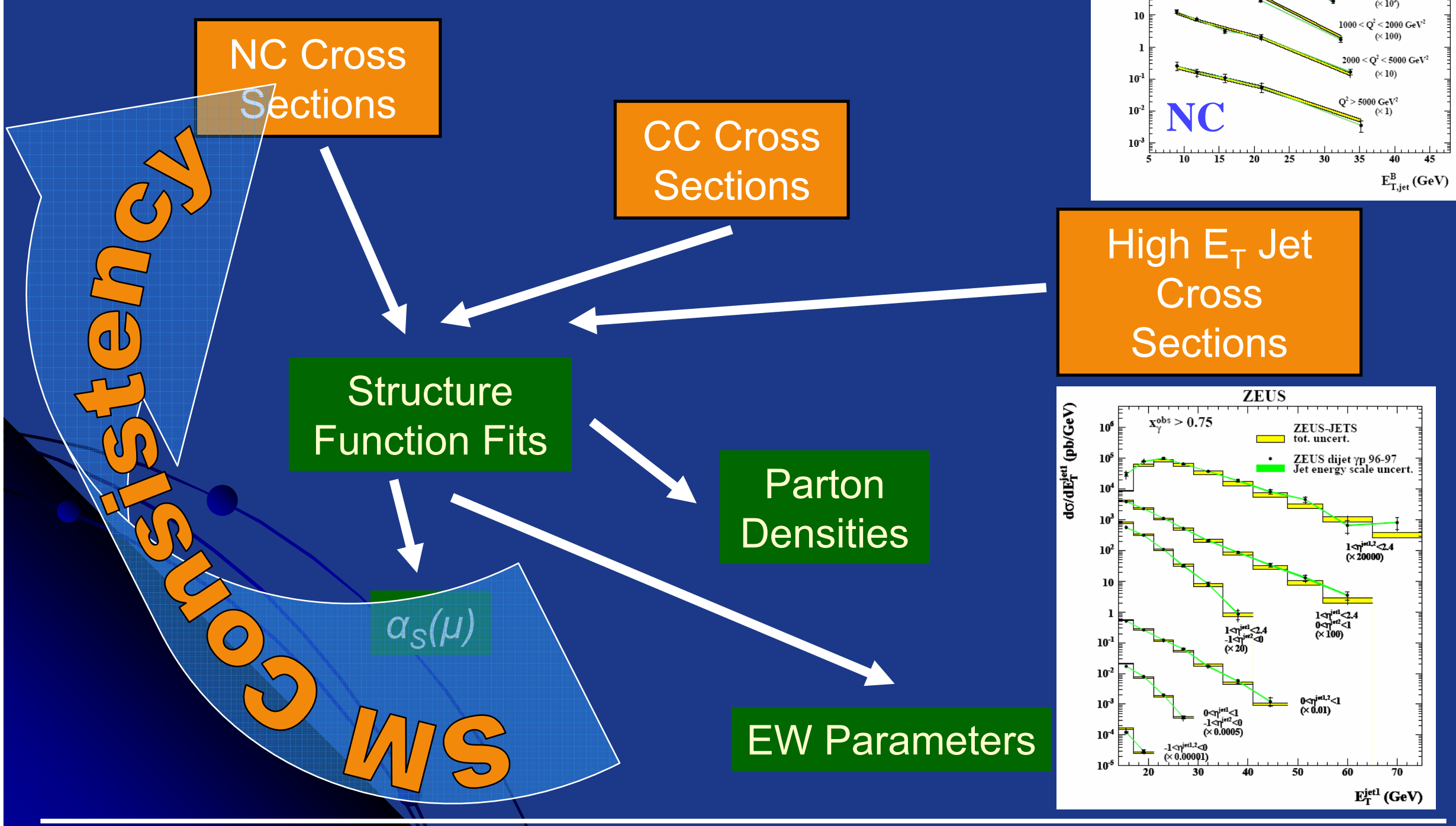




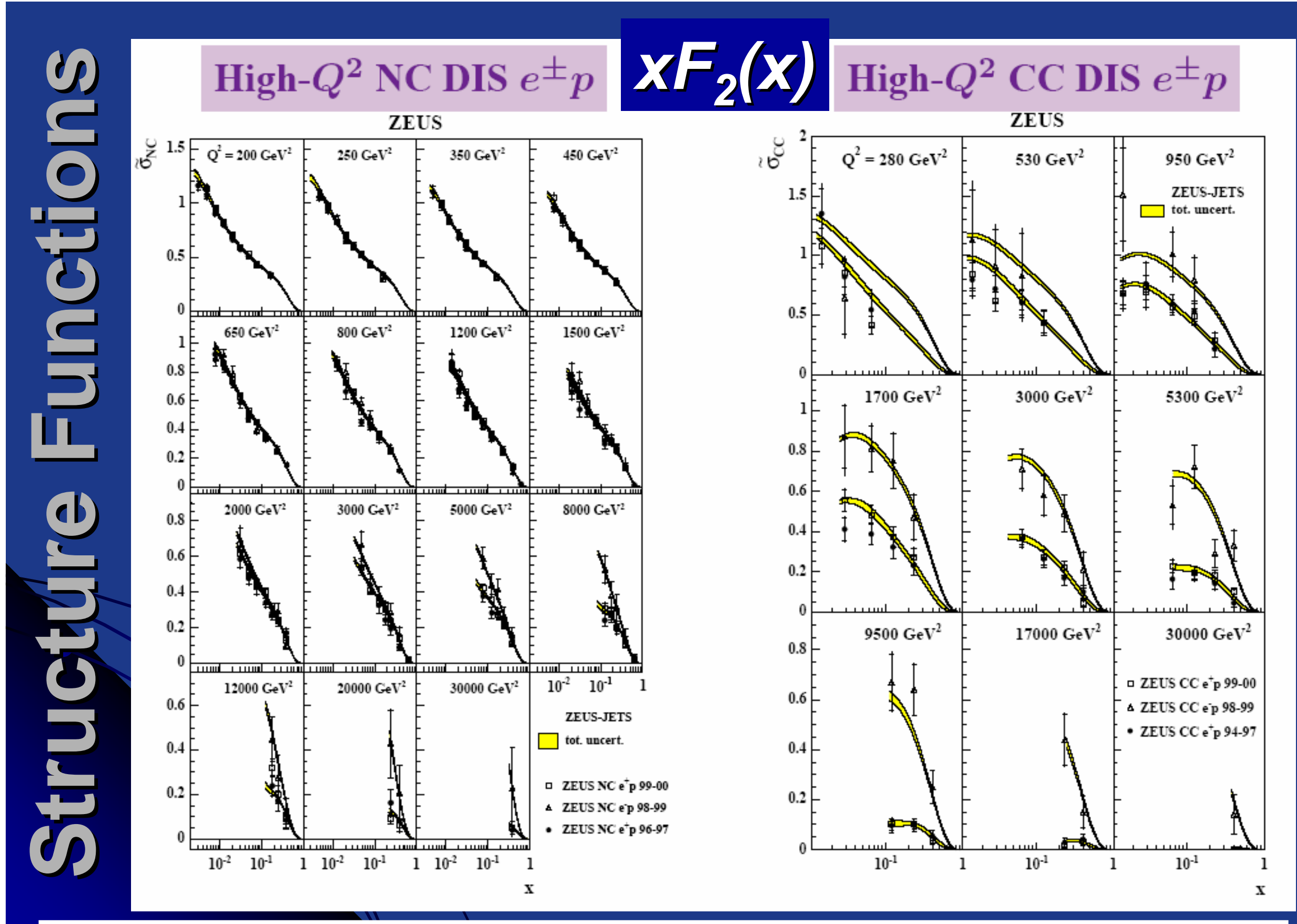




\section{NC cross section @ very high x}

\section{ZEUS}
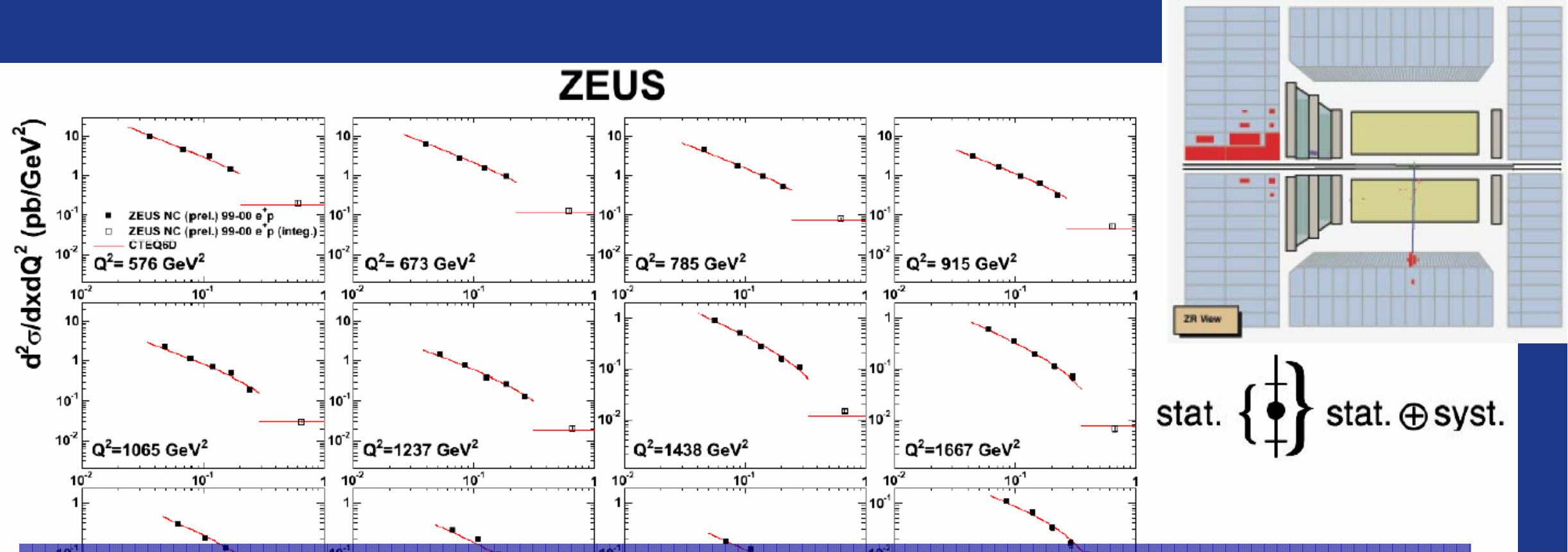

stat. $\left\{\begin{array}{l}\ddagger \\ q\end{array}\right.$ stat. $\oplus$ syst.

\section{High precision measurement up to $x_{i}=1$}

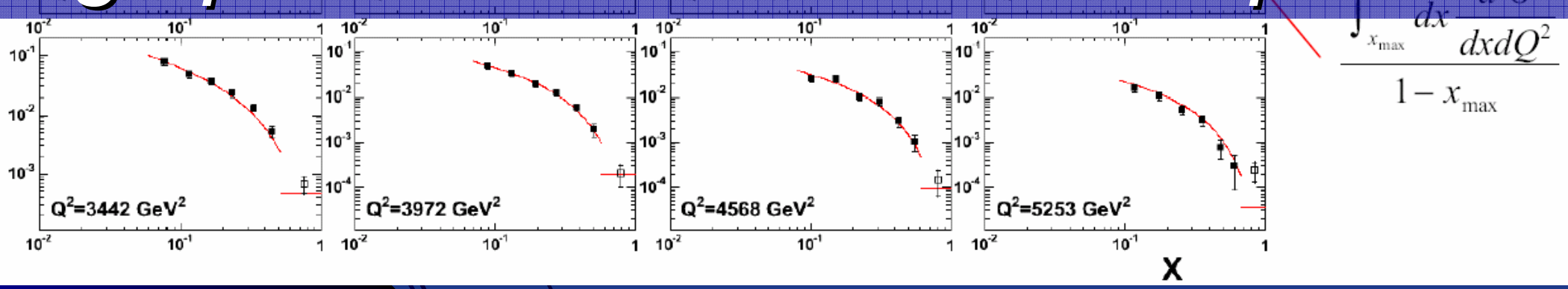




\section{$x F_{3}(x)$}

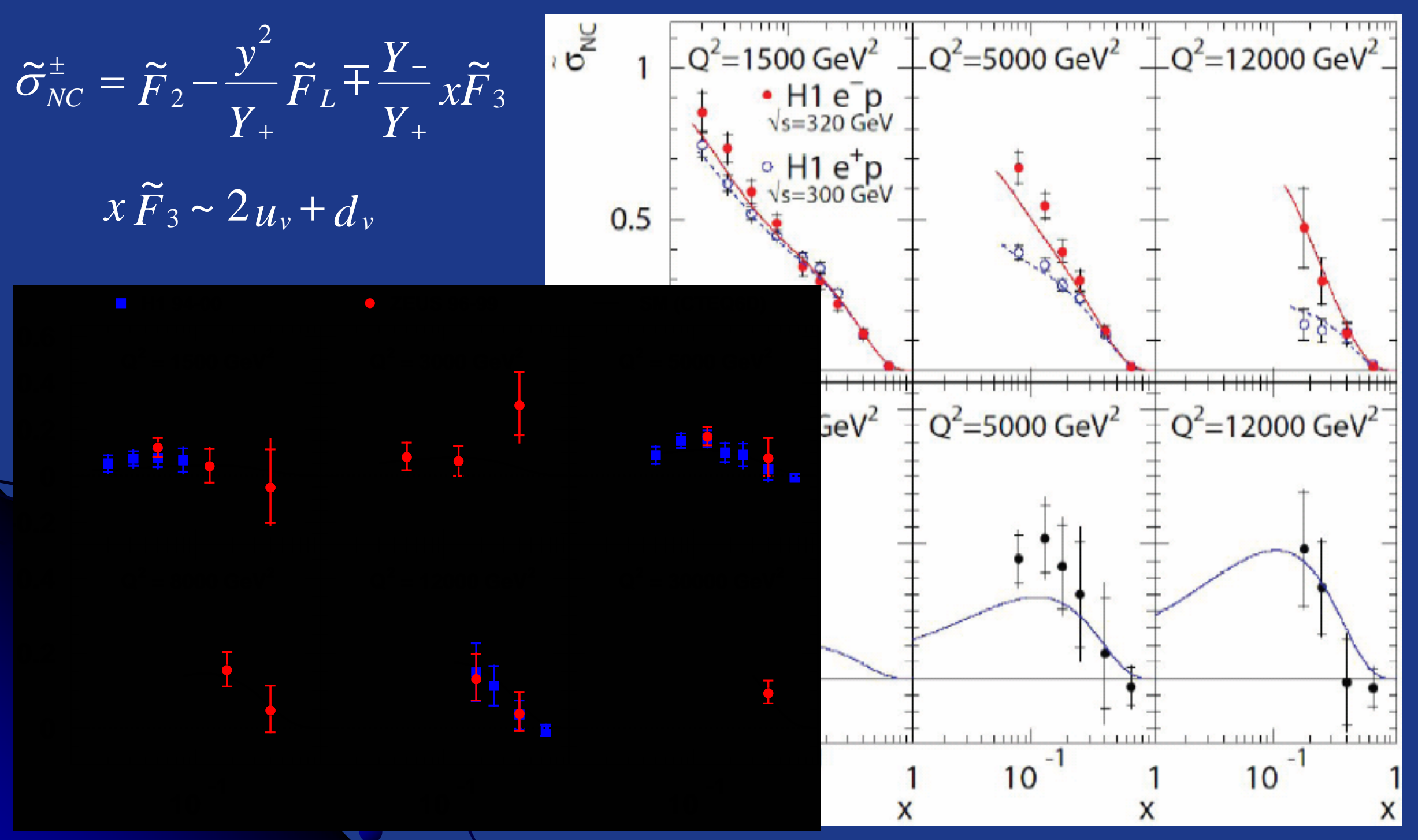




\section{Charm and beauty in the proton?}
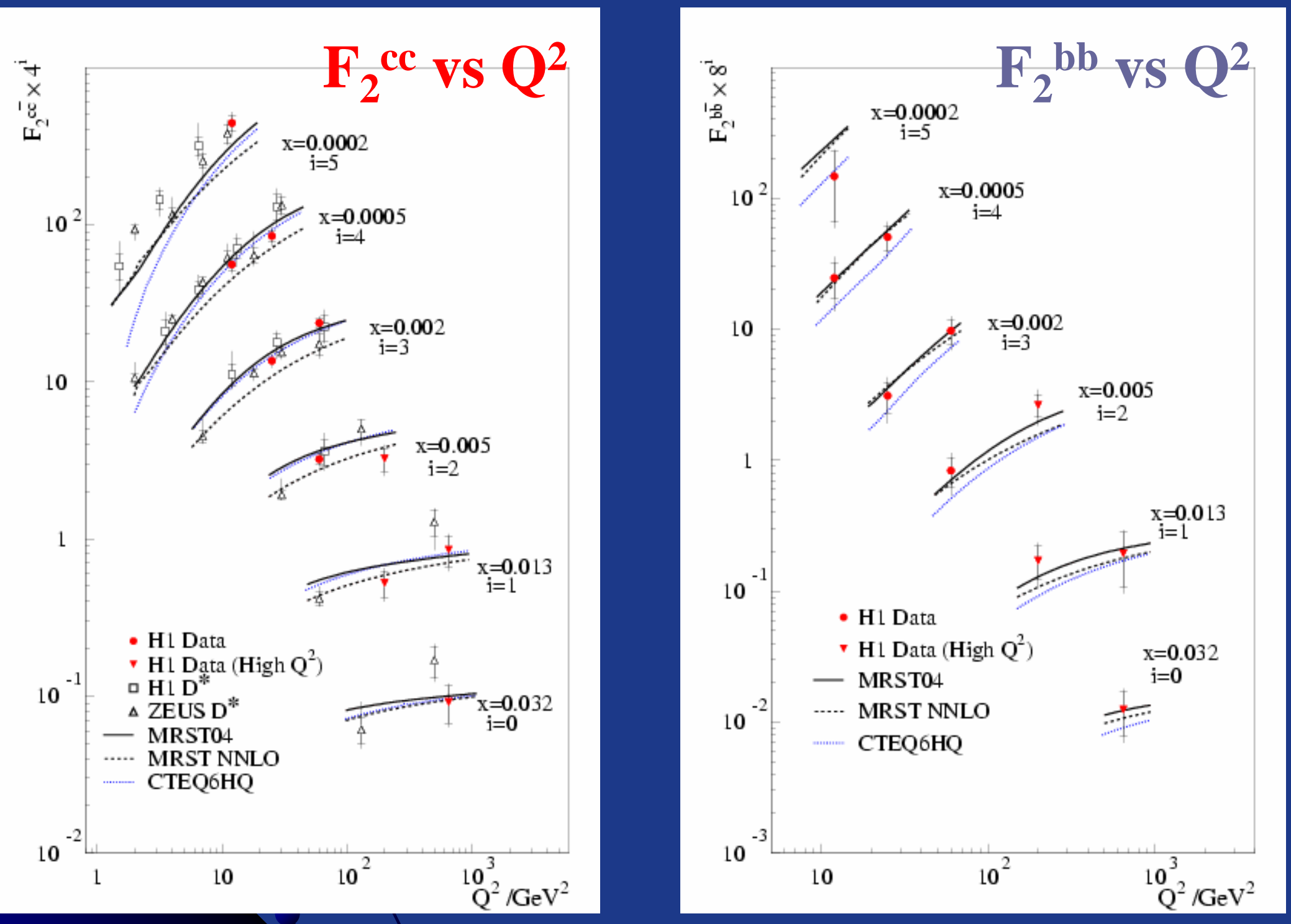


\section{$X F_{2}^{b}(x)$ and $X F_{2}^{c}(x)$}
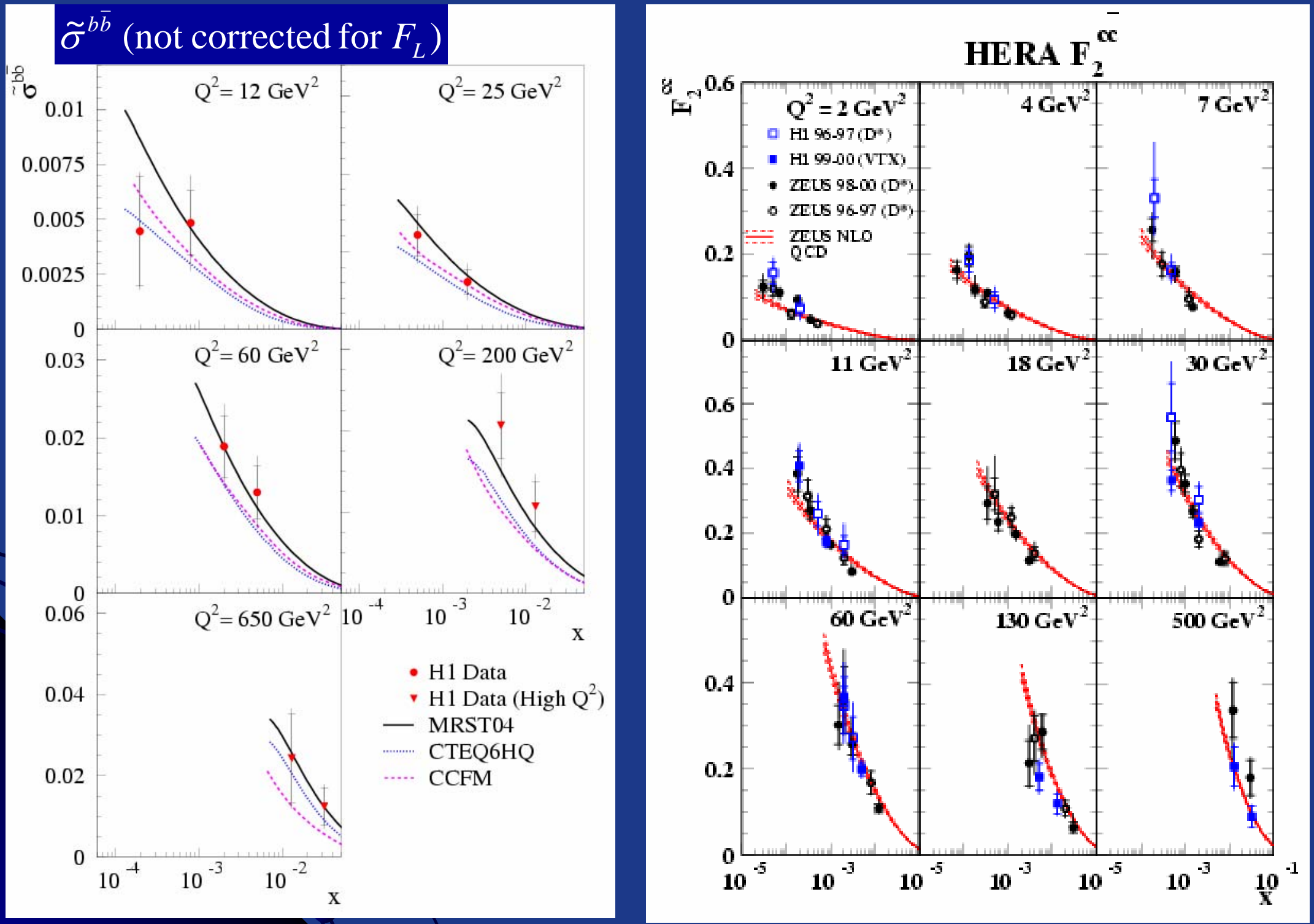


\section{Parton Densitjes}
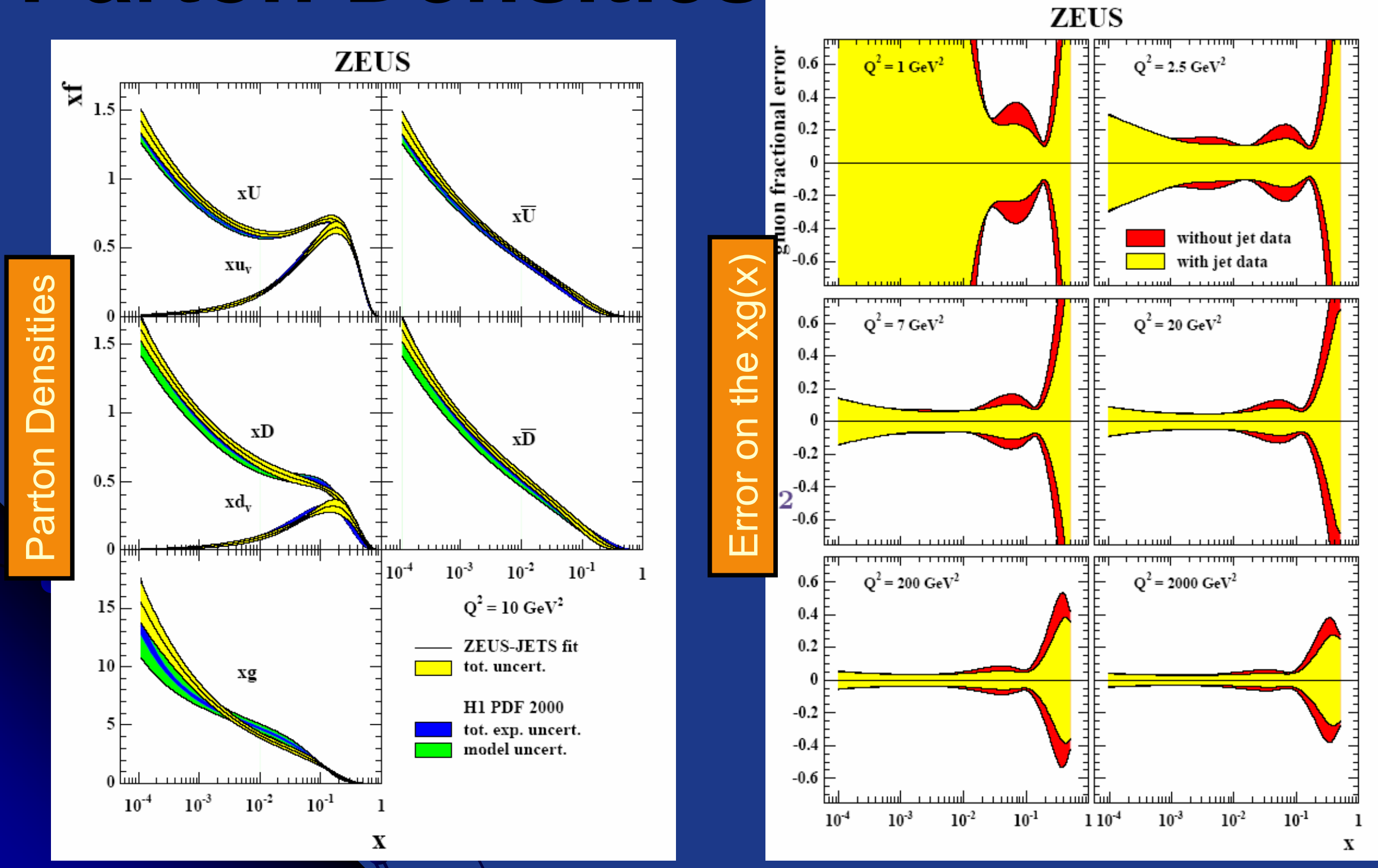


\section{HERA Parton Densities @ LHC}

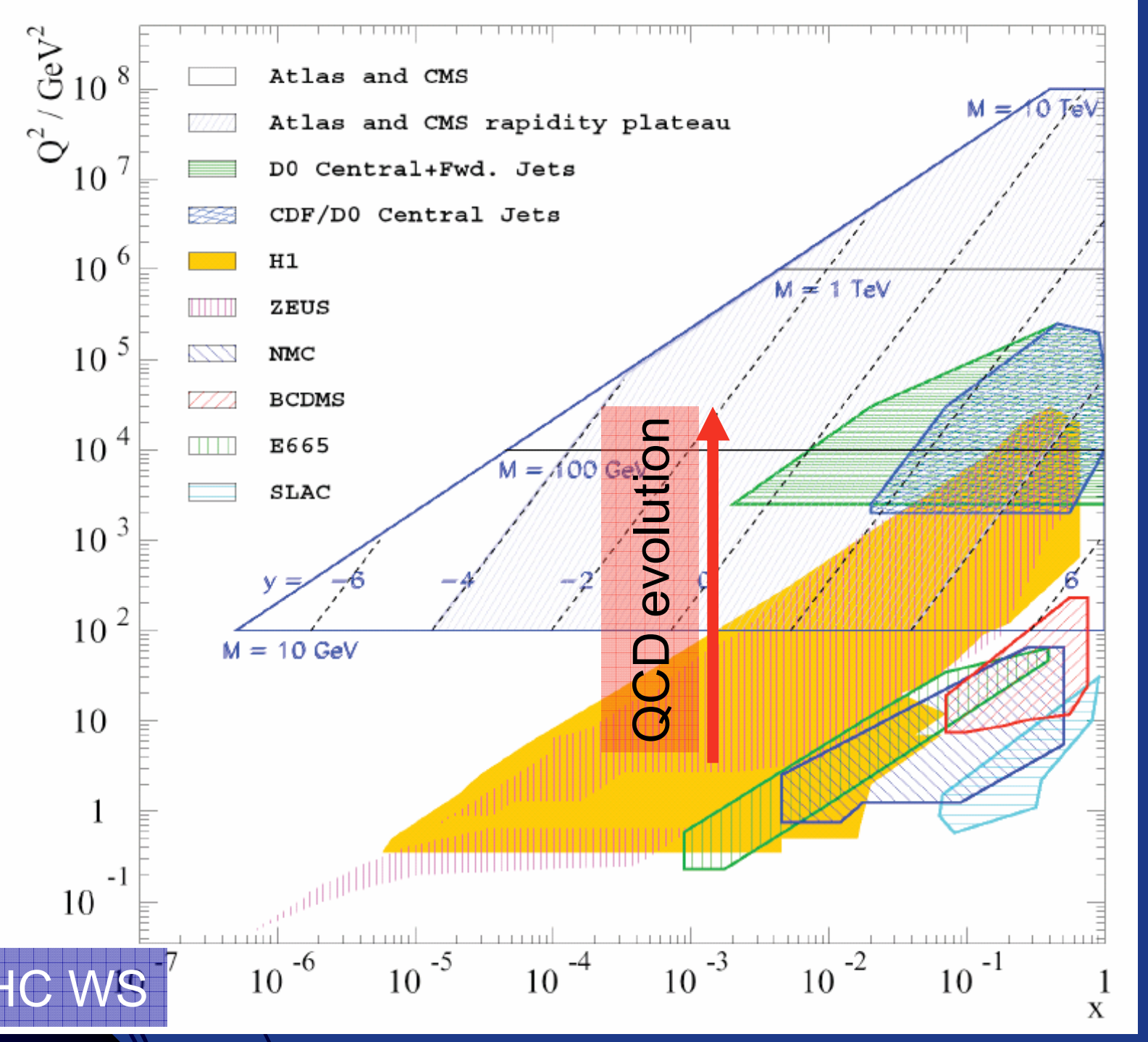

Ref: HERA-LHC WS

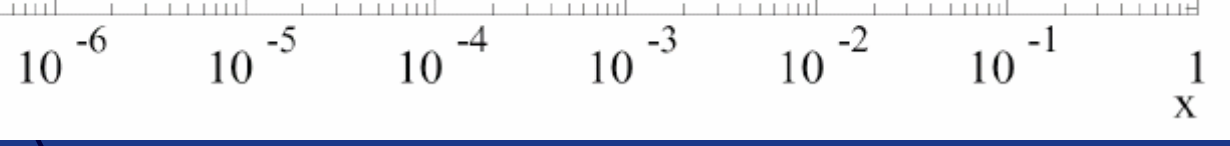




\section{$\alpha_{S}\left(M_{Z}\right)$}

- Simultaneous determination of $a_{s}\left(M_{z}\right)$ and PDFs

- Due to inclusion of jet cross sections $a_{S}\left(M_{Z}\right)$ and PDFs are NOT strongly correlated

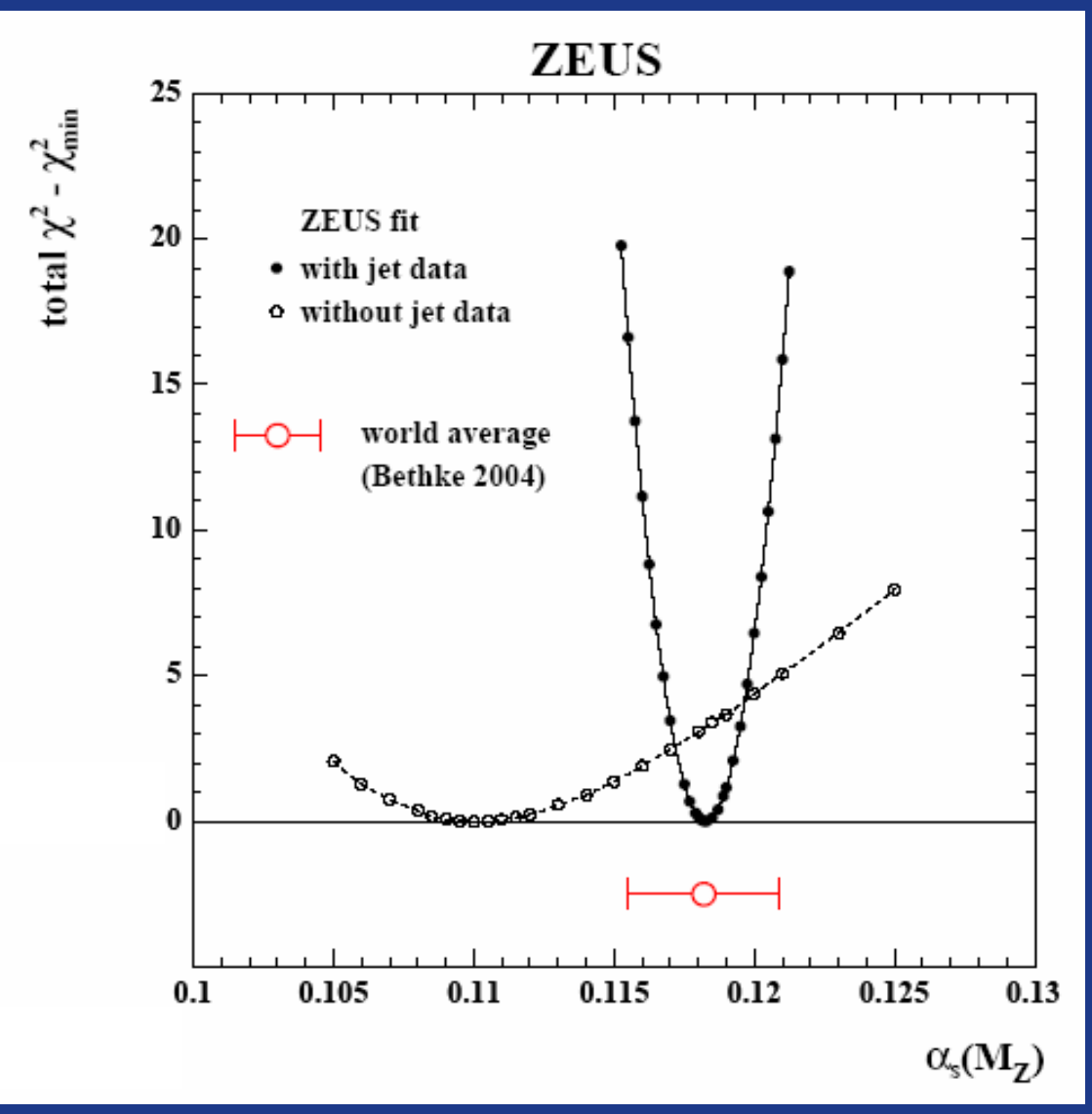

$$
\begin{array}{r}
\alpha_{s}\left(M_{Z}\right)=0.1183 \pm 0.0007 \text { (uncorr.) } \pm 0.0022 \text { (corr.) } \\
\quad \pm 0.0016 \text { (norm.) } \pm 0.0008 \text { (model) }
\end{array}
$$




\section{Precision of $\alpha_{S}\left(M I_{Z}\right) @$ HERA}

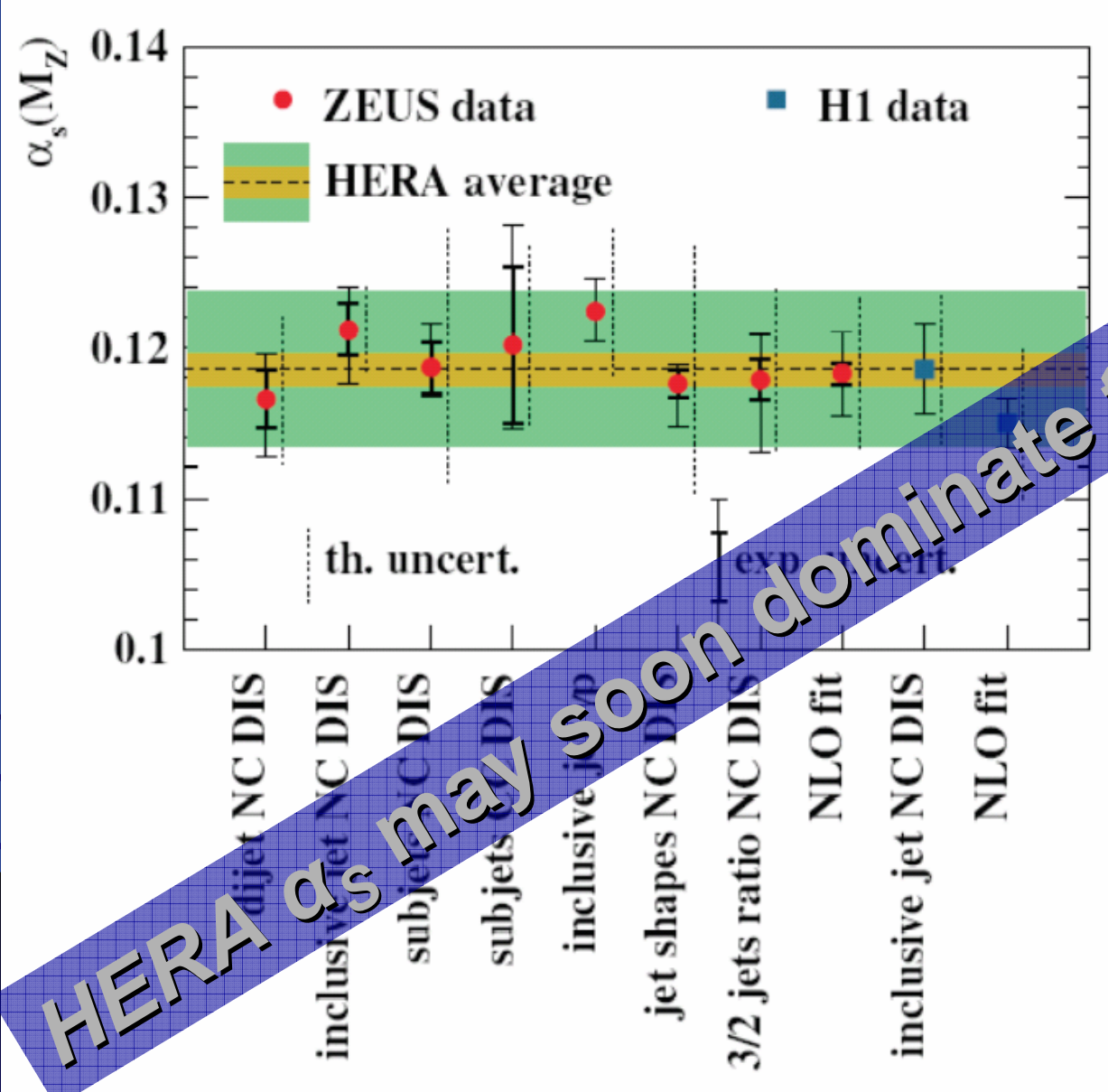

$\operatorname{HERA}($ prel. $)-\alpha_{s}\left(M_{Z}^{2}\right)=0.1186 \pm 0.0011(\exp ) \pm 0.005($ thy $)$

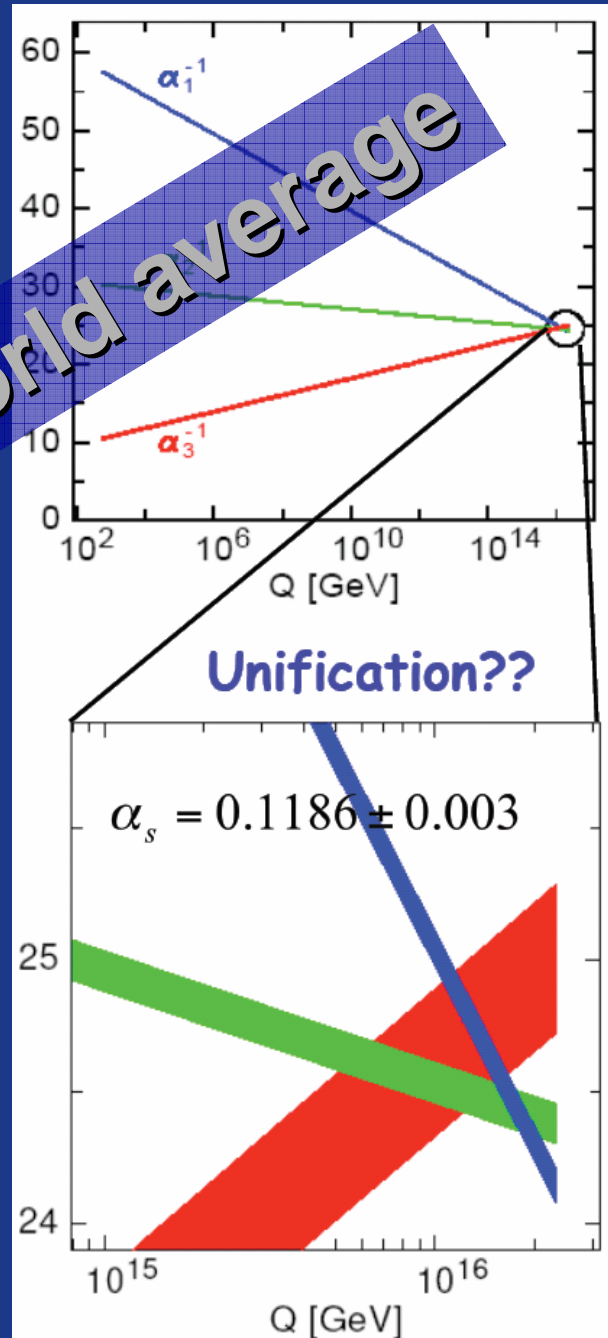

hep-ph/0407067 B.Allanach ... P.Zerwas 


\section{Light quark couplings}
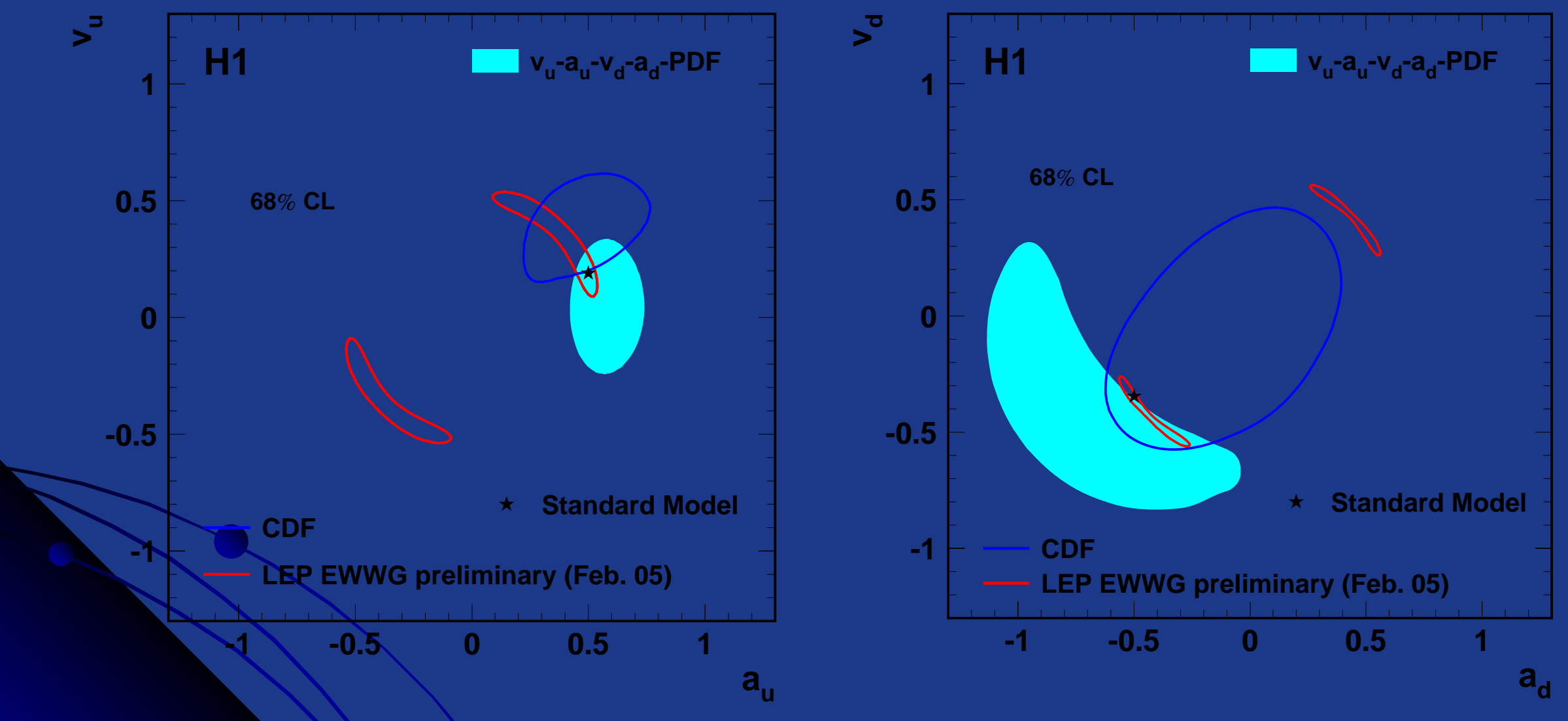

From H1 PDF fits including additional EW parameters 


\section{W Propagator Mass \& Coupling $\mathrm{G}_{F}$}

- HERA space-like $\left(q^{2}<0\right)$ mass vs. Tevatron/LEP timelike $\left(q^{2}>0\right)$ one

- SM relationship between $M_{W}$ and $G_{F}$ not used
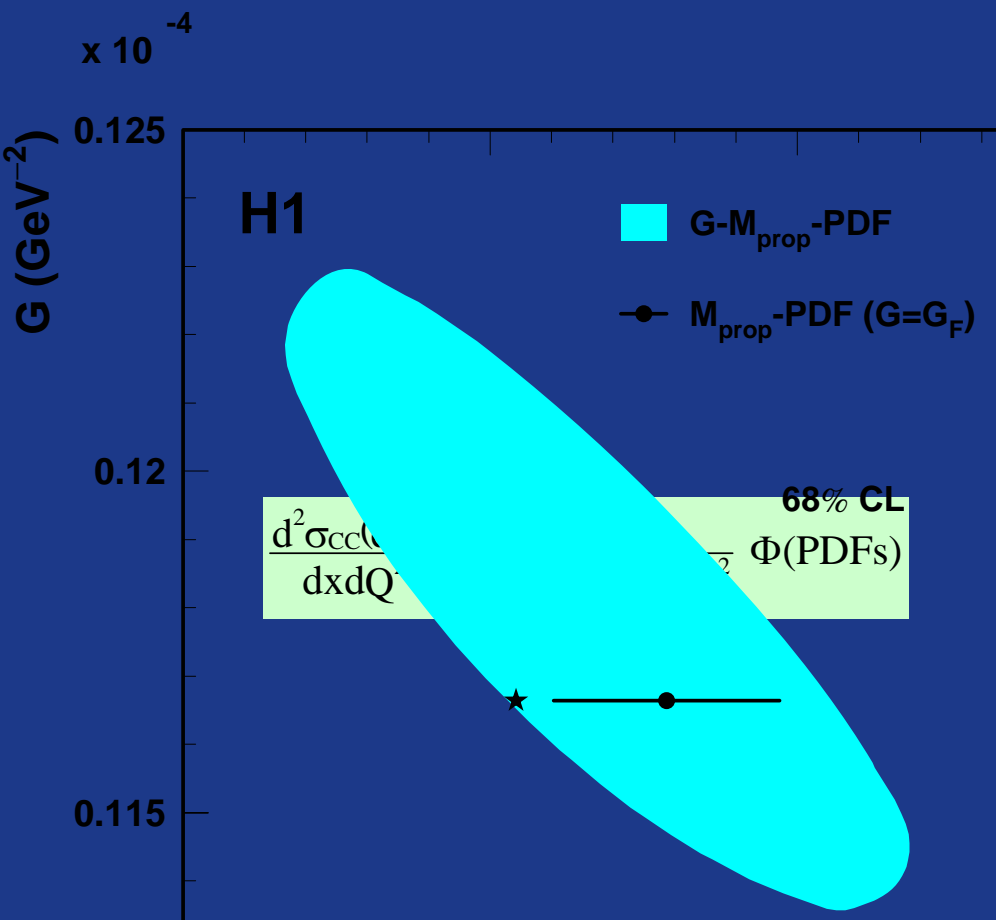

$$
M_{W}=82.87 \pm 1.83(\exp )_{-0.16}^{+0.30}(\bmod ) \mathrm{GeV}
$$




\section{$M M_{W}$ and $M_{t}$ in the SM}

- W mass value:

$\mathrm{M}_{\mathrm{W}}=80.786 \pm 0.205_{\exp } \mathrm{GeV}$

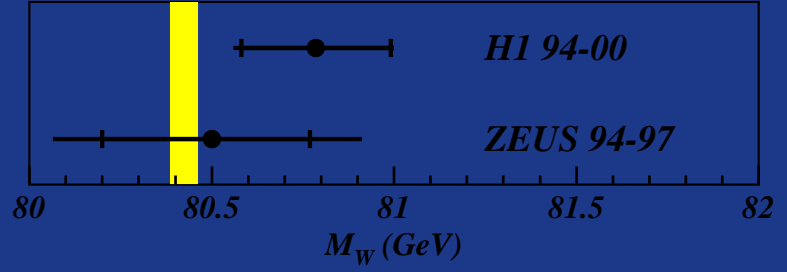

+ the world average $\mathrm{M}_{\mathrm{Z}}$

$\rightarrow$ indirect determination of

$\sin ^{2} \theta_{\mathrm{W}}=1-\mathrm{M}_{\mathrm{W}}{ } / \mathrm{M}^{2} \mathrm{Z}$ $=0.2151 \pm 0.0040_{\exp }$

- Top quark mass: $\mathrm{m}_{\mathrm{t}}=104 \pm 44_{\exp } \mathrm{GeV}$

$\Rightarrow$ First determination in DIS at EW scale

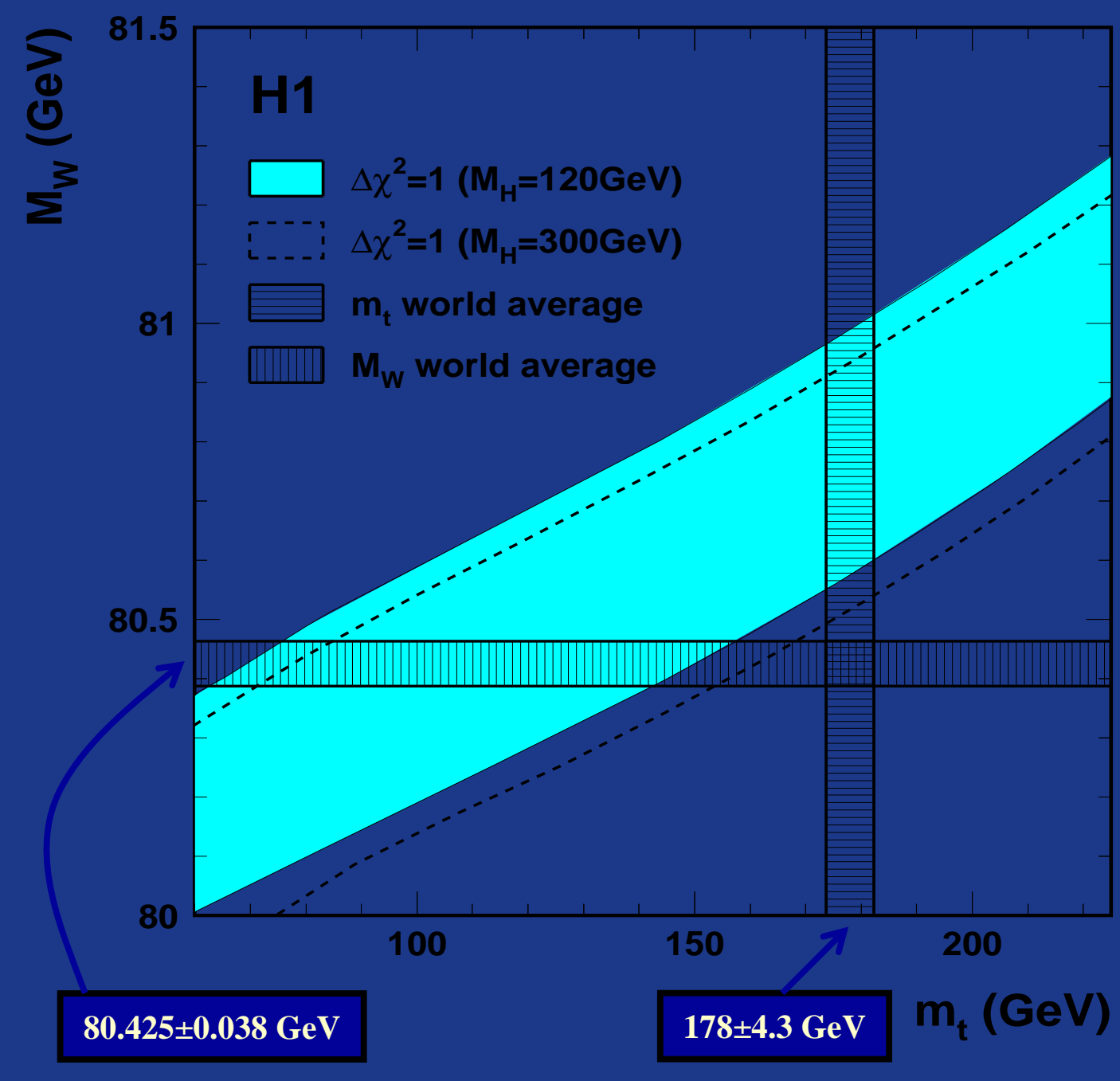




\section{Beyond the SM}




\section{Are quarks "elementary"?}

- Form factor measurement at $\mathrm{Q}^{2} \sim 40000 \mathrm{GeV}^{2}$

- Resolution $1 / \mathrm{Q} \sim 10^{-16} \mathrm{~cm}=0.001$ proton radius

- Compare measured to expected cross section

- Limits on quark size

oH1:

$R_{q}<1.0 \times 10^{-16} \mathrm{~cm}$

- ZEUS:

$R_{q}<0.85 \times 10^{-16} \mathrm{~cm}$

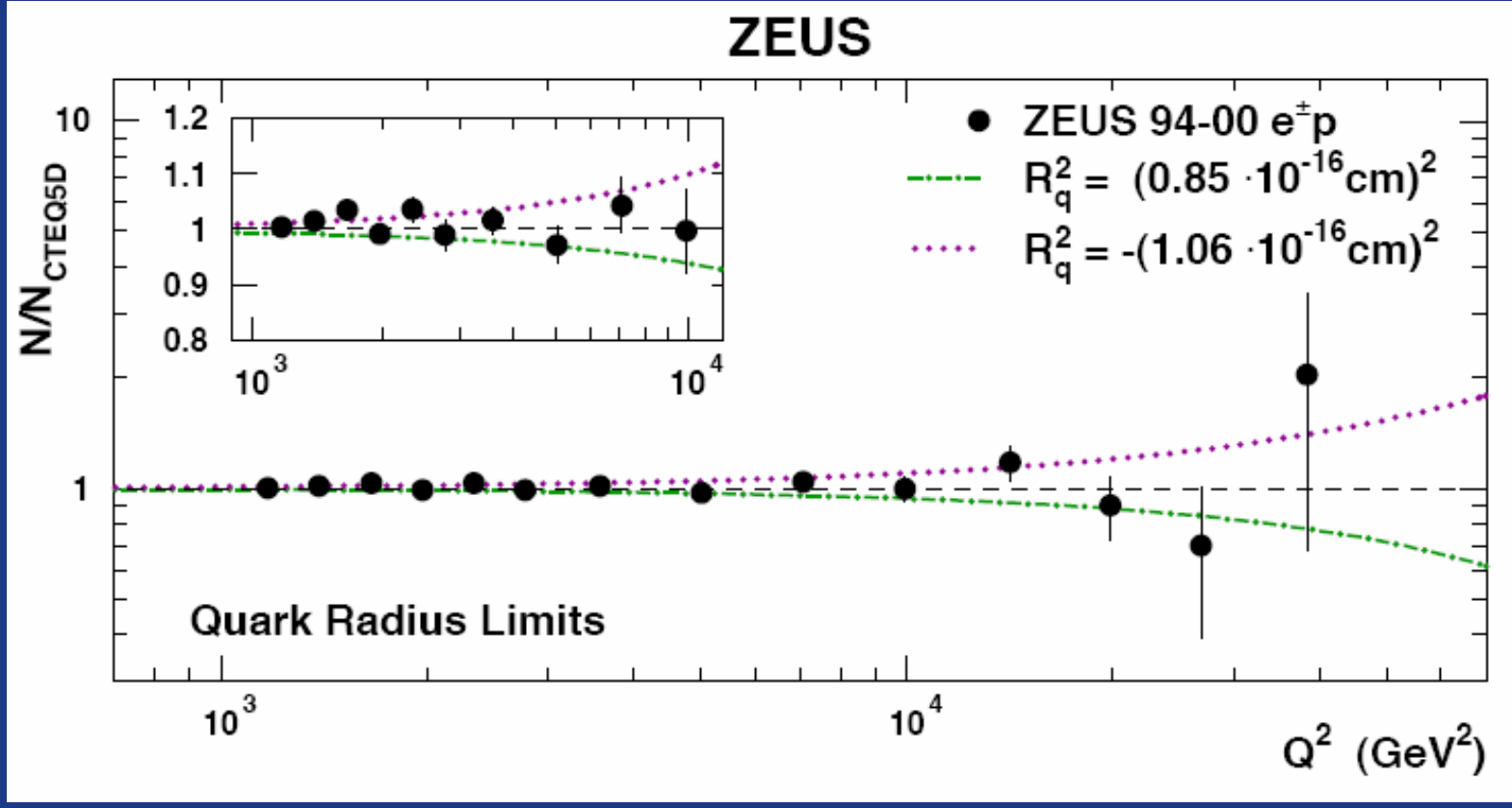




\section{Is there TeV scale physics?}

(Contact Interactions)

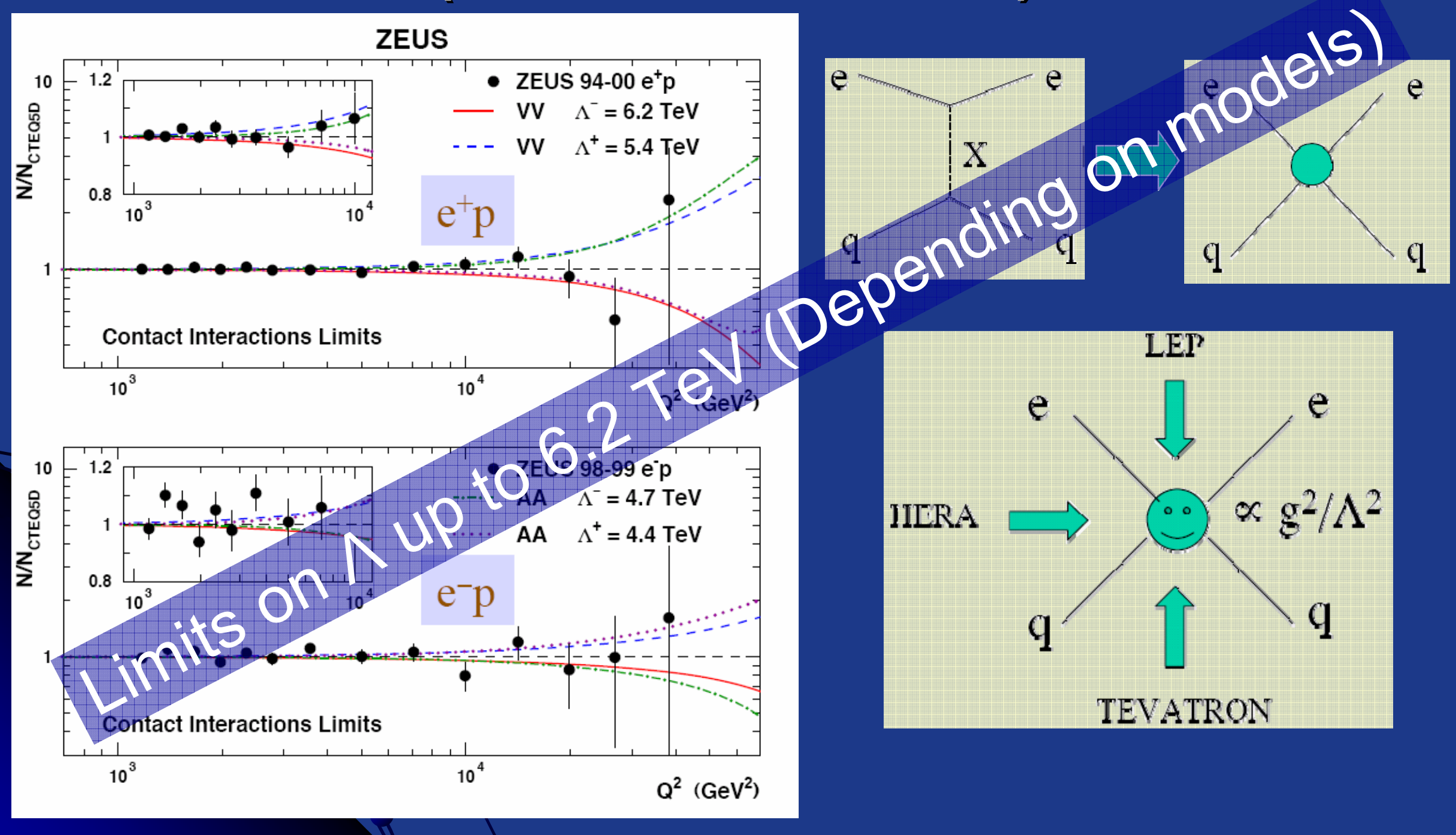


$\mathrm{q}$

$\lambda$ : Yukawa coupling

- HERA:

- s-channel eq fusion

- resonance in eq (vq) invariant mass in NC (CC) events

- no excess observed

O TEVATRON:

Q LQ pair production

- LEP2:

- virtual in $\mathrm{e}^{+} \mathrm{e}^{-} \rightarrow$ hadrons

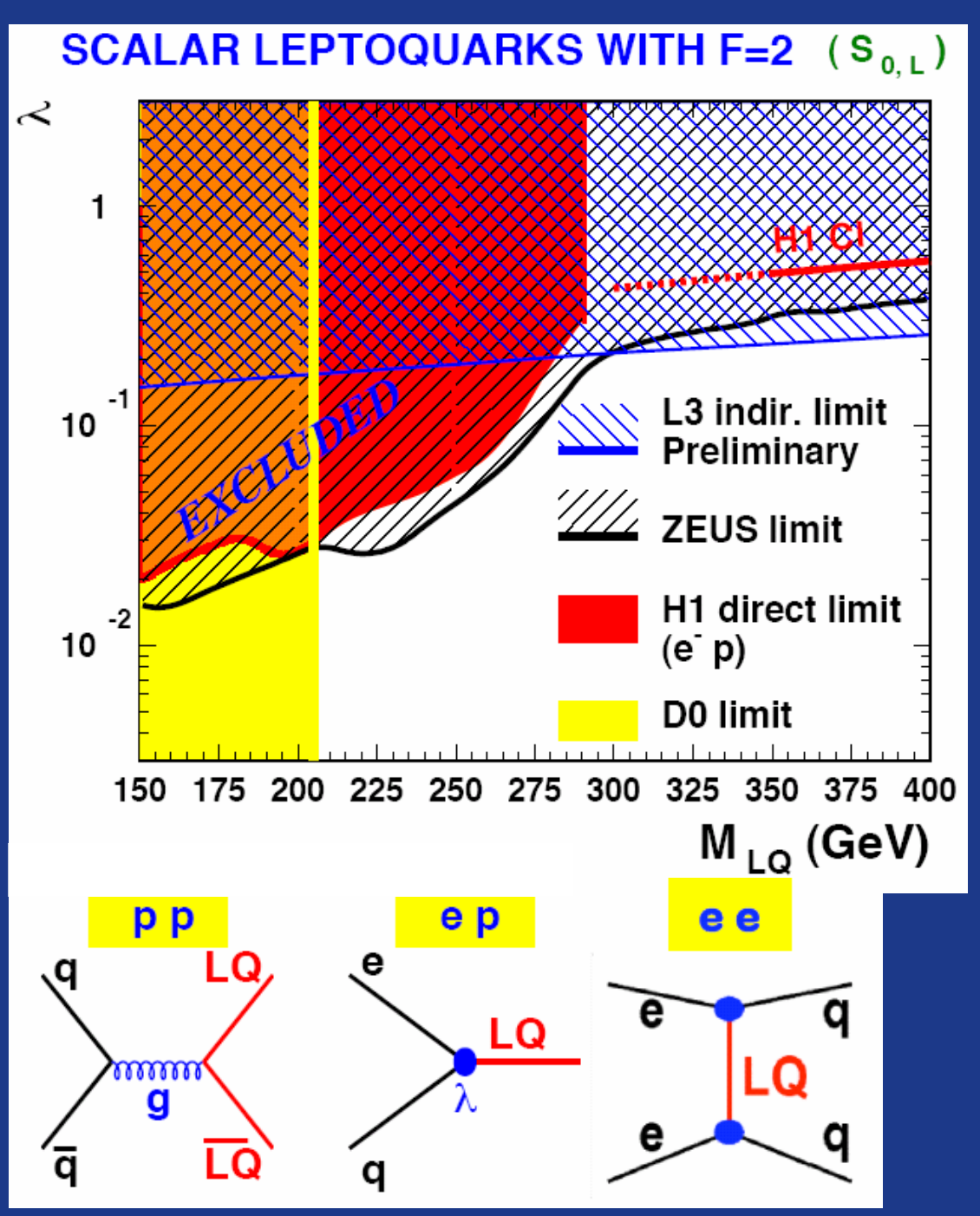




\section{Puzzle: High $p_{T}$ lepton events}

- Signature:

- lepton + missing $p_{T}+$ large $p_{T}($ had $)$

- Dominant SM process:

- on-shell $W$ production $(\sigma \sim 1 p b)$

- Excess seen in HERA I in H1 for $e / \mu$ and in ZEEUS for $\tau$

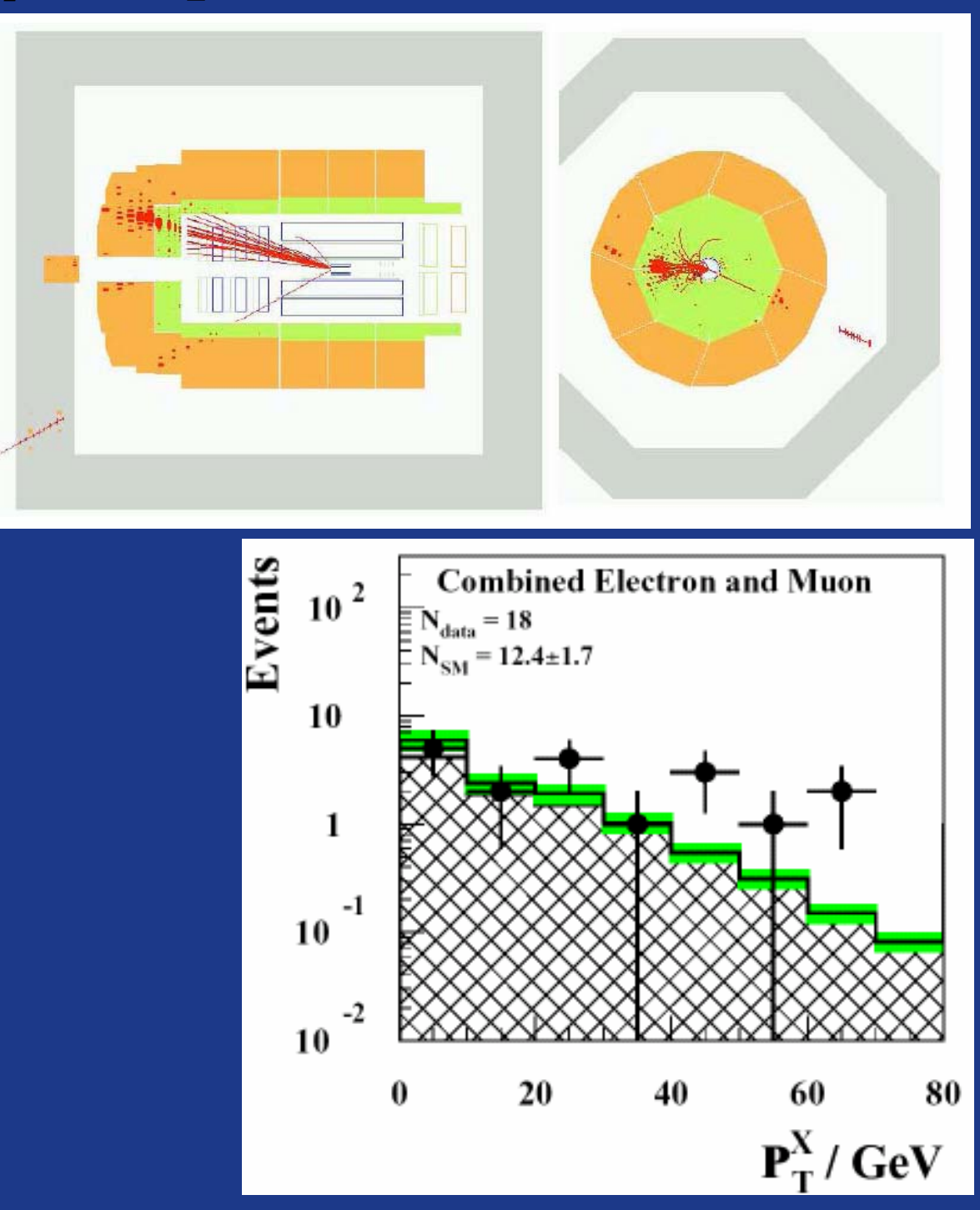




\section{High $\rho_{T}$ leptons: Latest numbers}

- H1 update with HERA II data $\left(74 \mathrm{pb}^{-1} \mathrm{e}^{ \pm} P\right.$ )

- 6 new electron events with $p_{T}(\mathrm{had})>25 \mathrm{GeV}$

- no new muon event

- overall excess in $e+\mu$ channel

- $\tau$ search at $\mathrm{H} 1$

a no excess seen (HERA I only)

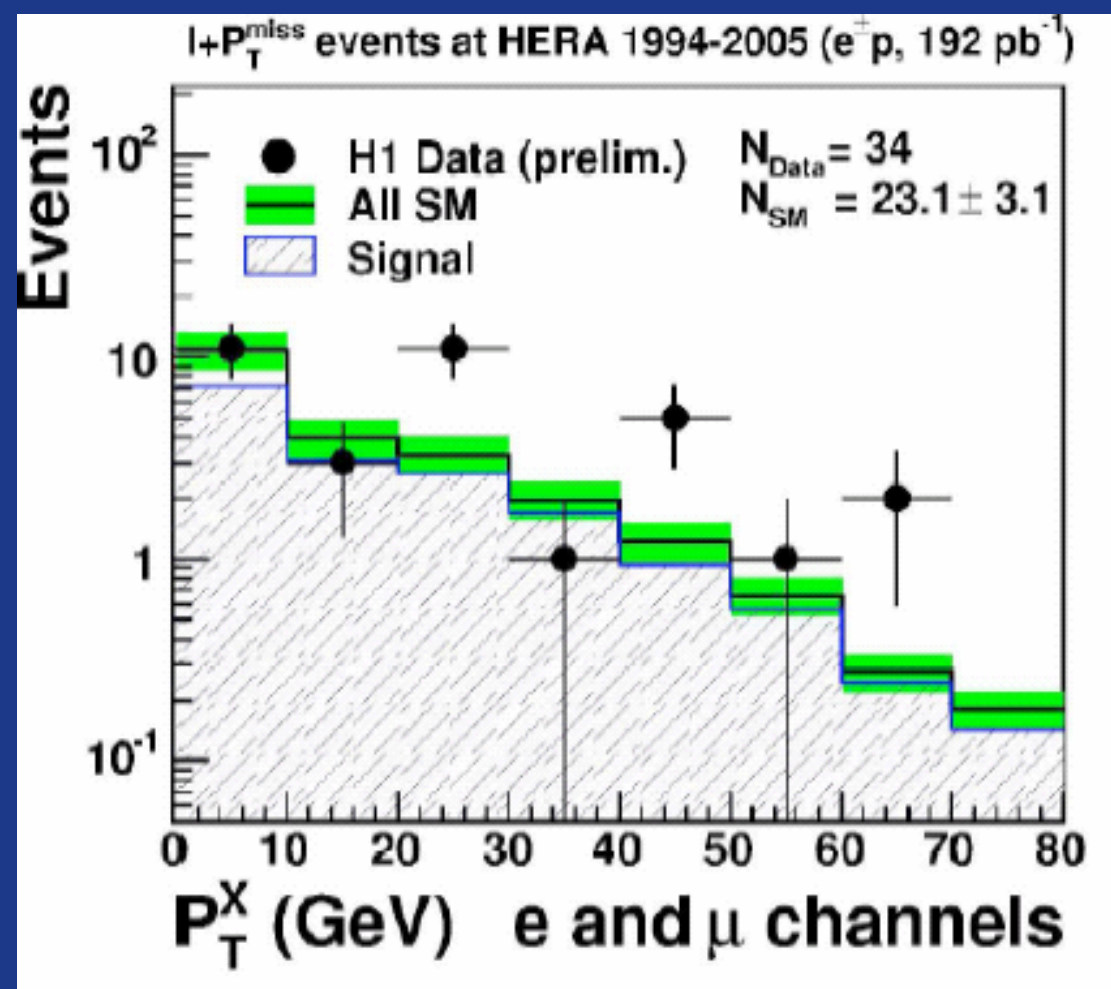

\begin{tabular}{|c|c|c|c|}
\hline () & $\begin{array}{c}\text { Electron } \\
\text { obs./exp. (W) }\end{array}$ & $\begin{array}{c}\text { Muon } \\
\text { obs./exp. (W) }\end{array}$ & $\begin{array}{c}\operatorname{Tau}^{\Phi} \\
\text { obs./exp. (W) }\end{array}$ \\
\hline All $P_{T}{ }^{x}$ & $25 / 18.3 \pm 2.5(70 \%)$ & $9 / 4.8 \pm 0.8(85 \%)$ & $5 / 5.8 \pm 1.4(15 \%)$ \\
\hline $\mathrm{P}_{\mathrm{T}}^{\mathrm{x}}>25 \mathrm{GeV}$ & $11 / 3.0 \pm 0.6(81 \%)$ & $6 / 3.0 \pm 0.6(86 \%)$ & $0 / 0.5 \pm 0.1(49 \%)$ \\
\hline
\end{tabular}




\section{Summary and Conclusions}

- HERA QCD results on structure functions, pdfs and $\alpha_{s}$ are becoming me most precise ...

- ... but HERA is not only a QCD machine:

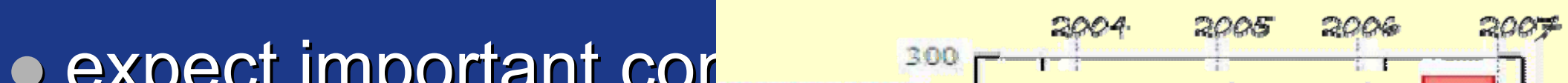
prect ing precision measurem $\frac{\text { Luminosity }}{\mathrm{pb}^{-1}}=200$

dexcellent search pot

- ... the really good st

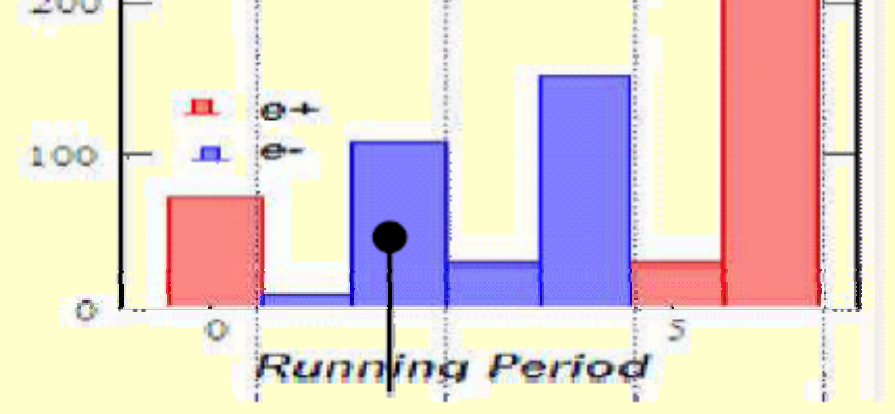

C. Gwenlan We are here... 